\title{
Fiber-associated Lachnospiraceae reduce colon tumorigenesis by modulation of the
}

4 Ana S Almeida ${ }^{1 *}$, Tam T T Tran ${ }^{1, \mathfrak{E}}$, Tarini S. Ghosh ${ }^{1}$, Celine Ribiere ${ }^{1}$, Cathriona Foley ${ }^{2}$, Lisa A

5 Azevedo ${ }^{1}$, Paola Pellanda ${ }^{1}$, Werner Frei ${ }^{1}$, Cara M Hueston ${ }^{1}$, Raju Kumar ${ }^{4}$, Burkhardt Flemer ${ }^{1}$, , Inês

6 Sequeira ${ }^{4}$, Micheal O'Riordain ${ }^{3}$, Fergus Shanahan $^{1}$, Paul W. O’Toole ${ }^{1, *}$

$8{ }^{1}$ APC Microbiome Ireland and School of Microbiology, University College Cork, Cork, Ireland

$9 \quad{ }^{2}$ Munster Technological University, Cork, Ireland

$10{ }^{3}$ Department of Surgery, Mercy University Hospital, Cork, Ireland

$11{ }^{4}$ Institute of Dentistry, Barts and the London School of Medicine and Dentistry, Queen Mary University

12 of London, London, UK

13 * Correspondence to a.almeida@ucc.ie or pwotoole@ucc.ie

${ }^{£}$ Current address: University of Science and Technology of Hanoi, Vietnam Academy of Science and

Technology, Hanoi, Vietnam 


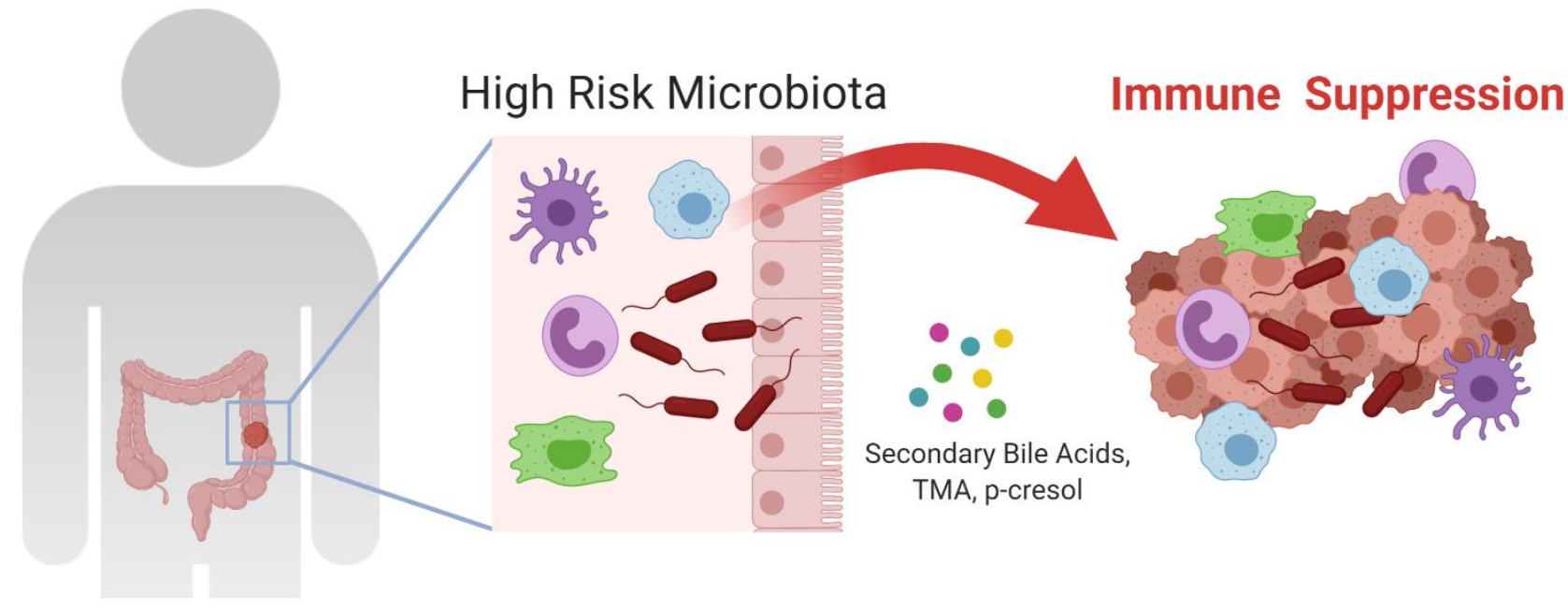

\section{Low Risk Microbiota}

Immune Activation

Proposed model of how the high-risk Pathogen and low-risk Lachnospiraceae CAGs differentially modulate the tumor immune response.

Short Title: Lachnospiraceae reduce colon tumorigenesis 


\section{Abstract}

Patients with colorectal cancer (CRC) harbor gut microbiomes that differ in structure and function from those of healthy individuals, suggesting this altered microbiome could contribute to tumorigenesis. Despite increasing evidence implicating the gut microbiome in CRC, the collective role of different microbial consortia in CRC carcinogenesis is unclear. We have previously described these consortia as co-abundance groups that co-exist at different abundance levels in the same patient. Here, we report that tumor biopsy tissue from patients with a "high-risk" Pathogen-type microbiome had a different immune transcriptome and immune cell infiltrate from those with a "low-risk" Lachnospiraceae-type microbiome. Transplantation from patients of the two fecal microbiome types into mice with an orthotopic tumor differentially affected tumor growth and the systemic anti-tumor immune response. The differences in tumor volume and immunophenotype between mice receiving the high-risk and the low-risk microbiome correlated with differences in the engrafted human microbial species and predicted microbiome-encoded metabolites in the two groups. Of twelve taxa whose abundance in recipient mice led to increased tumor onset, seven corresponded with differentially abundant taxa in a global dataset of $325 \mathrm{CRC}$ patients versus 310 healthy controls. These data suggest that the enrichment for a Lachnospiraceae-type configuration of the gut microbiome may influence colon cancer progression and disease outcome by modulating the local and systemic antitumor immune response.

Keywords: Colorectal cancer, gut microbiota, tumor microbiota, metagenomics, bacterial metabolites 


\section{Introduction}

Colorectal cancer $(\mathrm{CRC})$ is among the top three causes of global cancer-related mortality and the incidence continues to increase worlwide ${ }^{1}$. Risk factors for CRC include age, a diet low in fiber and rich in red meat and fat, and chronic inflammation of the gastrointestinal tract ${ }^{2,3}$. All these factors are closely associated with altered composition and function of the gut microbiota. The gut microbiota from patients with polyps or CRC differs from that of healthy individuals ${ }^{4,5}$. However, whether this "dysbiosis" contributes to disease or is a consequence of cancer is not yet clear ${ }^{6}$. Differences in the gut microbiota also explain why some patients with cancer benefit from new cancer immunotherapies while other patients do not ${ }^{7}$.

Studies to date in mice have indicated several ways by which specific bacterial species might impact CRC development. Proposed CRC-promoting bacteria or "oncobacteria" include Fusobacterium nucleatum $^{8,9}$, genotoxic (pks+) Escherichia coli ${ }^{10,11}$, enterotoxigenic Bacteroides fragilis ${ }^{12}$, Streptococcus gallolyticus ${ }^{13}$, and Peptostreptococcus anaerobius ${ }^{14}$. Despite the increasing evidence implicating the gut microbiota in $\mathrm{CRC}$ development, the role of microbial community-driven pathogenicity as distinct from single taxa still needs to be elucidated.

Bacteria can directly contribute to the development of CRC by the release of genotoxic factors ${ }^{15}$, production of carcinogenic metabolites ${ }^{16}$, or by interacting with the immune system ${ }^{17}$. Infiltration by immune cells heavily impacts clinical outcome in $\mathrm{CRC}^{18}$. Numbers of intratumoral $\mathrm{T}$ cells correlate positively with better $\mathrm{CRC}$ outcomes, including disease-free and overall survival ${ }^{19}$. However, the role of innate immune cells in CRC is less clear. Under certain circumstances, neutrophils and macrophages release radical oxygen species and nitrous oxide, which can potentially cause genomic damage in colonic epithelial cells ${ }^{20}$. The presence of specific immune cells in the tumor microenvironment, as well as the release of lymphocyte-attracting chemokines and cytokines, can be modulated by the microbiota, either directly or through their metabolites ${ }^{6}$. Understanding how specific microbiota taxa and their metabolites differentially modulate the host immune response has important clinical implications for CRC patients, including diagnosis, and potentially also treatment. In our previous analyses of the gut microbiome in CRC patients, we used clustering methodologies to identify six co-abundance groups or CAGs (i.e. co-abundance associations of genera) ${ }^{4}$, which we 
82 subsequently simplified to five $\mathrm{CAGs}^{21}$. A single subject harbored multiple CAGs but their relative

83 abundance differed between CRC patients and healthy controls, with three of the 5 CAGs being more

84 abundant in CRC patients. Tumor biopsies from patients whose microbiome was dominated by these

85 CAGs showed differential expression of 18 genes involved in inflammation and CRC progression ${ }^{4}$,

86 suggesting a possible microbiome influence on tumor development. To test this, here we used human-

87 into-mice fecal microbiota transplants from patients with adenomas or adenocarcinomas with a "high

88 risk' or a 'low risk' microbiota. We show for the first time that the presence of a Pathogen CAG or a

89 Lachnospiraceae CAG microbiome differentially affected tumor progression. Distinct bacterial taxa

90 correlated with tumorigenesis, different metabolic pathways, and divergent systemic immune

91 responses that correlated with tumor volume. 


\section{Results}

Different global transcriptome in human colonic tumors with the Pathogen CAG and the

\section{Lachnospiraceae CAG}

To investigate if bacterial CAGs present in CRC patients may shape the tumor immune profile and affect the host response, we recruited a cohort of 32 treatment-naive patients with either adenomas or adenocarcinomas and undergoing resection surgery (Fig. 1A, Table S1). None of these patients had received antibiotics or probiotics in the 3 months before surgery, nor been treated with chemotherapy or radiation therapy. Samples were collected from different mucosal sites, including from the tumor (or adenoma) and nearby healthy tissue. Using the approach we previously described ${ }^{4,22}$, we profiled the abundance of the 5 different bacterial CAGs in the 32 patients and named them according to the dominant genera in each group -- Bacteroidetes, Lachnospiraceae,

Pathogen, Prevotella, and Rumminococcus (Figs. 1B, S1, S2; Table S2). As expected, the majority of the patients had a higher abundance of the Pathogen CAG compared to the other CAGs.

We selected 12 representative patients whose microbiome was dominated by either the Pathogen CAG $(n=6)$ or the Lachnospiraceae CAG $(n=6)$, together with biopsies from 10 healthy controls ${ }^{4}$, and subjected them to RNA seq analysis. Patients with adenomas or CRC were chosen accordingly to their microbiota profile and independently of tumor staging, size or location, although the majority of the tumors were located in the rectum or sigmoid colon (Table S1). First, we identified 21 (FDR $<0.1)$ and an additional 518 ( $p$ value $<0.05$ ) genes significantly differentially expressed between Pathogen and Lachnospiraceae enriched tumors, with 268 upregulated in the Pathogen CAG and 271 upregulated in the Lachnospiraceae CAG tumors (Fig. 1C, Tables S3 and S4). Next, we identified significantly differentially expressed genes (DEGs) between healthy controls and the Pathogen CAG $(11,520)$, and healthy controls and the Lachnospiraceae CAG $(11,205)$ enriched tumors (Fig. 1C, Table S3). To further refine the transcriptomic differences found, we identified genes uniquely elevated in Pathogen or Lachnospiraceae dominated tumors compared to healthy controls and performed unsupervised hierarchical clustering (Fig 1D). This revealed that the two microbiota CAGs were associated with distinct host transcriptome differences. Pathway level analysis indicated that the greatest transcriptional changes were related to upregulation of the pathways involved in epithelial- 
mesenchymal transition, inflammatory response, angiogenesis, and interleukin-6 (IL-6) JAK STAT3 signaling in Pathogen-enriched tumors versus Lachnospiraceae-enriched tumors (Fig. S3, Table S4).

We next focused on genes associated with the host immune response. Relative to healthy controls, Lachnospiraceae enriched tumors from patients with adenomas or CRC showed upregulated genes associated with immune signatures that were not different in Pathogen enriched tumors. These included antigen presentation genes (HLA-C, HLA-B, TRPC4AP, PSMD2, ULBP3), TCR signaling genes (MAP3K8, CBLC, CD3E, MAP3K14), chemokines predictive of increased survival in CRC ${ }^{23,24}$ (CXCL11, CXCL13), innate immune genes (IL1A, IL33, NLRP6) and the inhibitory receptor PD1 $(P D C D 1)$ (Fig. 1F). In contrast, genes associated with monocytes or monocytic myeloid-derived suppressor cells (MDSCs) signature and signaling were differentially upregulated only in Pathogenenriched tumors compared to healthy controls (CSF1R, CD163, CSF1, CXCR2), as well as the inhibitory receptor CTLA4 (Fig. 1E). These differentially expressed immune genes were also significant after testing for confounding by tumor site, tumor stage, and lymph node involvement (Table S5).

To functionally interpret these results, we used the CIBERSORTx (ell type Identification By Estimating Relative $\underline{\text { Subsets }} \underline{\text { Of known }} \underline{\mathrm{R} N A}$ Iranscripts) method $^{25,26}$ to deconvolute the gene expression data and estimate immune cell composition in CRC tumors. Pathogen CAG enriched tumors had a significantly higher estimated abundance of monocytes and lower estimated abundance of lymphocytes compared to healthy controls, whereas there were no statistical differences between Lachnospiraceae CAG tumors and healthy controls (Fig 1G, Fig. S4, Table S4). Specific leukocyte subset analysis identified a significant increase of M2 macrophages in Pathogen enriched tumors compared to Lachnospiraceae enriched tumors, but not M0 or M1 macrophages (Fig. 1H). Altogether these results suggest that the abundance of different microbiota taxa is associated with specific host gene expression changes, including differential upregulation of several immune pathways and recruitment of specific immune cells to the tumor microenvironment.

\section{A Lachnospiraceae-dominant microbiome reduced tumor growth in a pre-clinical mouse} model of CRC 
Although previous studies have determined that certain bacterial species can influence tumor progression and response to therapy ${ }^{6,7}$, the mechanism(s) by which specific bacterial clusters present in $\mathrm{CRC}$ patients might influence tumor progression has not yet been determined. We therefore investigated the effect of the two different CAGs - Pathogen and Lachnospiraceae - on tumor development using the MC-38 orthotopic cancer mouse model ${ }^{27}$. This orthotopic model allows for the reliable and efficient study of tumors arising in immune-competent animals at the appropriate primary site $^{28}$. Germ-free (GF) mice were administered one of two representative human microbiota types, from donors selected based on their mucosal microbiota profile (Fig. 2A). We selected donor 1 (CRC044, female, T3N2b rectum adenocarcinoma) because their mucosal microbiota was mostly composed of the Pathogen and Prevotella CAGs (44.3\% and $11.4 \%$, respectively, Figs. 2A, S5, Table 1). We selected donor 2 (CRC056, male, adenoma with low-grade dysplasia) with a mucosal microbiota dominated by the Lacnhospiraceae CAG (41.3\%, Figs. 2A, S5, Table 1). After human fecal administration, we allowed 8-10 days for colonization and stabilization of the human microbiota ${ }^{29}$ and then injected the MC-38 colorectal cancer cells in the rectal submucosa of these mice, as previously described ${ }^{30}$ (Fig. 2B). Mice that received the Pathogen CAG microbiota developed larger tumors than mice that received the Lachnospiraceae CAG microbiota (Fig. 2C). Surprisingly, mice that received the Lachnospiraceae CAG developed very small tumors, even smaller when compared to the GF control group, and 3 out of 12 mice did not develop any tumors $(p=0.022$ and $p=0.063$, two independent experiments) (Fig. 2C). We also observed that mice that received the Pathogen CAG displayed a much higher level of variability in their tumor volume than the Lachnospiraceae CAG-receiving group.

We investigated the recipient mouse fecal microbiota composition using $16 \mathrm{~S}$ rRNA gene amplicon (16S) sequencing. As expected, $\beta$-diversity analysis showed a donor-specific signature in each recipient group $\left(R^{2}=0.79, p=0.001\right)$ (Fig. 2D). Similar to $\beta$-diversity, $\alpha$-diversity analysis of the recipient mice resembled donor microbiota diversity, with mice in the Pathogen CAG group harboring a less diverse fecal microbiota than the Lachospiraceae CAG group (Fig. S6, S7, Table S6). Strikingly, the differences observed in tumor growth between the Pathogen CAG and the Lachnospiraceae CAG mouse groups were significantly correlated with the mouse microbiota profiles 
at both weeks 2 (after human microbiota administration but before MC-38 cells injection) and week 5 (three weeks after MC-38 cells injection, endpoint) $\left(R^{2}=0.25, p=0.006\right)$ (Figs. 2E). This finding strengthened the causal link between the engrafted microbiota composition and tumor onset in the MC-38 mouse model. Notably, mice that received the Pathogen CAG were also characterized by significantly higher microbiome composition variability from the corresponding donor compared to those that received the Lachnospiraceae CAG, suggesting differences in the resilience or community cohesion of the two different microbiome types (Fig. S8).

\section{Distinct pathobiont and commensal taxa strongly correlate with tumorigenesis and predict} tumor growth

To identify with greater taxonomic resolution the bacterial taxa that were associated with tumorigenesis, and their respective functions, we performed shallow shotgun sequencing ${ }^{31}$ on mouse fecal samples collected at weeks 2 and 5. Using Spearman's correlation analysis between the abundance of the bacterial species present at each time point and the tumor volume at week 5 , we identified several bacterial species that significantly correlated with tumor volume (Figs. 3, S9). Bacterial species that were positively correlated with tumor growth (referred to henceforth as 'tumor positive') were consistently present in higher abundance in mice that received the Pathogen CAG compared to mice that received the Lachnospiraceae CAG, while species negatively correlated with tumor growth ('tumor negative'), showed the opposite trend. The taxa identified by 16S sequencing as tumor-positive or tumor-negative largely overlapped between the two experiments performed (Fig. S10), but the shallow shotgun sequencing data performed for the second replicate (experiment 2) afforded much greater taxonomic resolution and correspondingly more taxa. At week 2 , we identified 23 taxa that showed significant association with tumor volume (Fig. 3). Interestingly, 8 out of these 23 were also associated with tumor volume at week 5 (Fig. 3). These included four tumor-negative bacterial taxa, 2 of which belonged to the Lachnospiraceae family (Coprococcus comes and Ruminococcus lactaris), and 4 tumor-positive taxa (Ruminococcus obeum, Clostridium hathewayi, Flavonifractor plautii, and Coprococcus sp ART55 1). In addition to these eight, there were 28 additional bacterial taxa whose abundance was significantly associated with tumor volume at week 5 
only (Fig. 3). Tumor-positive taxa included Bacteroides sp (B. fragilis, B. salyersiae, B. faecis, $B$.

uniformis), Paraprevotella sp., Clostridium boltae, and Desulfovibrio piger. Bacterial species that were negatively associated with tumor growth at week 5 included Akkermansia muciniphila, Barnesiella intestinihominis, Alistipes sp., and Bifidobacterium sp., (B. longum and B. pseudocatenulatum) (Fig. 3).

To check the clinical relevance of these microbiome-tumor associations, we examined the abundance of these taxa in six patient-control paired studies whose CRC-associated microbiome had been shotgun sequenced (Global Reference CRC cohort, as described in the methods) ${ }^{32-37}$. We calculated the correlation between these bacterial taxa and CRC by comparing the effect size between CRC patients $(n=352)$ and healthy individuals $(n=310)$ across the six studies (Fig. 3, Table S7). We found

7 out of 12 tumor-positive taxa at week 2 to be significantly more abundant in CRC patients when compared to healthy subjects (Fig. 3). These included several Clostridia reported as pathobionts $(C$. hylemonae, C. symbiosum, C. asparagiforme, C. hathewayi, and Clostridiales bacterium 1-7-47FAA), along with $F$. plautii and Bacteroides caccae. In contrast, while 5 of the tumor-negative taxa were enriched in the healthy individuals, only $C$. comes and $R$. lactaris (Lachnospiraceae family) reached significance. Interestingly, B. longum and B. pseudocatenulatum (week 5) were also present in significantly lower abundance in CRC patients compared to healthy individuals, while $B$. fragilis, $B$. salyersiae, and C. boltae were significantly more abundant in CRC patients Some associations between bacterial taxa and tumor volume (e.g. Bilophila wadsworthia and Bilophila unclassified) were not confirmed in the human dataset. This suggests that these bacterial species might be donorspecific and not involved in tumor progression, at least in the MC-38 mouse model. Another example was $R$. obeum which showed a positive association with tumor volume at both weeks 2 and 5 , but a negative association in the Global Reference CRC cohort. However, within the Pathogen CAG group, R. obeum showed a negative association with tumor volume, being in much higher abundance in mice that developed the smallest tumors (Fig. S11).

229 To explore if the dynamic shifts in microbial composition observed before tumor development (week 2) and afterward (week 5) could be a predictor of disease, we used Random-Forest (RF) classifier models to identify the bacterial species at the different time-points that predicted tumor volume. We 
232 found that the bacterial species at week 2 had a significantly higher predictive value compared to the

233 bacterial species found at week $5(p<0.006)$ (Figs. 4A, S12). We also confirmed this effect was not

234 due to a loss of species over time (data not shown). This result suggests that the transplanted taxa 235 that correlated with tumor volume after 2 weeks of engraftment were better indicators of final tumor 236 volume than the 5-week taxa, indicating that early microbe-cell interactions were pivotal for 237 determining cancer progression.

The tumorigenic high-risk metagenome is characterized by detrimental metabolic pathways associated with CRC pathogenesis

The results obtained up to this point in our investigation strongly suggested a causal link between the microbiome type and tumorigenesis. To explore potential mechanisms, we investigated the metabolic functions associated with the two microbiota types. For this purpose, we identified a validated set of predicted metabolic capabilities that were 1) differentially abundant between the Pathogen CAG and Lachnospiraceae CAG mouse groups, and 2) differentially abundant in CRC patients versus healthy individuals in the Global Reference CRC cohorts (refer to Fig. S13 for methodology). As expected, Pathogen CAG and Lachnospiraceae CAG microbiomes were associated with different metabolic pathways (Fig. S14). Differences in the predicted microbiome production of secondary bile acids (i.e. lithocholic acid (LCA) and deoxycholic acid (DCA)), trimethylamine (TMA), p-cresol, acetone, and ammonia were positively associated with the Pathogen CAG (Fig. 4B). Similarly, increased consumption of multiple short-chain fatty-acids (SCFAs), mainly driven by $D$. piger, was associated with the high-risk Pathogen CAG, a trait that could lead to the depletion of available SCFAs (Fig. S15, Table S7). In contrast, the predicted production of several vitamins including pyridoxal phosphate, folate, and thiamine, was associated with the low-risk Lachnospiraceae microbiota, exclusively driven by B. longum and B. pseudocatenulatum (Fig. 4B, Table S8). Interestingly, pyridoxal, an active form of vitamin $\mathrm{B} 6$, has been associated with a $30-50 \%$ reduction in $\mathrm{CRC}$ incidence ${ }^{38}$.

257 A limitation of the above data was that these were inferred based on experimentally known production and consumption profiles derived from reference genomes and organisms. To investigate whether these metabolic functionalities were also encoded in the genome of the strains present in the mouse 
fecal samples, we profiled the genes for enzymes known to confer these functions, focusing on the production of secondary bile acids, and genes for choline to TMA production. For secondary bile acid synthesis (DCA and LCA), a group of the bai gene cluster (baiF, baiN, baiE, baiCD, and baiA) is known to be involved. Based on the gene family abundances obtained in the HUMAnN2 analysis, we first checked and compared the coverage of the bai gene cluster in the samples derived from the Pathogen and the Lachnospiraceae microbiome recipient mice (Fig. 4C). The high-risk Pathogen CAG had significantly greater coverage of the bai gene cluster compared to the Lachnospiraceae CAG indicating a significantly greater probability of this metabolic function being present in the former $(p<0.006)$. Similarly, the Pathogen CAG also had a significantly larger copy number of the CutC and CntA enzymes that catalyze the conversion of choline to TMA, further validating the results of the inferred metabolite profiling ( $p<0.02$; Fig. 4 C). In contrast, the low-risk Lachnospiraceae CAG had significantly higher copy numbers of the AtoD enzyme that catalyzes the formation of the antiinflammatory SCFAs - butyrate, propionate, and acetate - from Butyryl CoA, Propionyl CoA, and Acetyl CoA $(p<0.0004$ Fig. 4C).

\section{A Lachnospiraceae-type microbiome is associated with a strong systemic anti-tumor response}

To determine whether the difference in tumor growth rates in recipient mice could be related to host immunity, we investigated immune cell populations in the spleen of mice by flow cytometry. There were significantly more neutrophils $\left(C D 45^{+} \mathrm{CD} 11 \mathrm{~b}^{+} \mathrm{MHCll}-\mathrm{Ly} 6 \mathrm{G}^{+}\right.$Ly6 $\left.6^{\text {low }}\right)$, monocytes (CD45+CD11 b+MHCII-Ly6G-Ly6 ${ }^{\text {high }}$ ), macrophages (CD45+CD11 b+MHCII+), and dendritic cells (DCs) $\left(\mathrm{CD} 45^{+} \mathrm{CD} 11 \mathrm{~b}-\mathrm{MHCll}+\mathrm{CD} 11 \mathrm{c}^{+}\right)$in spleens from mice receiving the Pathogen $\mathrm{CAG}$ than in mice receiving the Lachnospiraceae CAG (Fig. 5A). In contrast, mice receiving the Lachnospiraceae CAG had multiple differences in the adaptive immune cell populations present in the spleen, including an increase in overall numbers of $\mathrm{CD} 3+, \mathrm{CD} 4+\mathrm{T}$ cells, and cytotoxic $\mathrm{CD} 8+\mathrm{T}$ cells, which is consistent with an active and effective anti-tumor immune response in these mice (Fig. 5B). There were also significantly more NK T cells $\left(\mathrm{CD}^{+}{ }^{+} \mathrm{CD} 335^{+}\right)$in spleens of mice receiving the Lachnospiraceae CAG than in mice receiving the Pathogen CAG, with no change in NK cells (CD3-CD335') (Figs. 5B, S16). 
288 These findings in the mouse model, together with the RNAseq analysis of human tumor tissue 289 suggesting a strong and differential regulation of a microbiota-dependent immune response prompted 290 us to characterize and quantify the immune infiltrate in the human biopsies using 291 immunofluorescence. There was an increase in tumor-infiltrating CD15+ neutrophils in Pathogen CAG 292 enriched tumors, while Lachnospiraceae CAG-enriched tumors had increased tumor-infiltrating total 293 CD3 $^{+}$T cells (Figs. 5C-D, S17). Together, these data demonstrate that a Lachnospiraceae-dominant 294 microbiome is associated with a strong local and systemic adaptive immune response, while a 295 Pathogen-enriched microbiome is associated with an immunosuppressive myeloid phenotype. 296 Collectively these results suggest that the abundance of particular taxa is associated with specific 297 host immune signatures that likely dictate tumor fate. 


\section{Discussion}

299 Although human studies have suggested a causal link between an oncogenic microbiota and 300 microbiota-induced immune response in CRC, there has been a lack of compelling causative 301 evidence to support this concept. Our study consolidates the microbial ecology aspect of this thesis 302 by showing that mice that received a human-derived Lachnospiraceae CAG microbiota developed much smaller orthotopic tumors than those receiving a Pathogen CAG microbiota. Based on our data and published studies we propose that the Pathogen-type microbiota, which is relatively overabundant in CRC patients ${ }^{4}$, induces the recruitment of neutrophils, myeloid-derived suppressor cells, and M2 macrophages, enhancing tumor growth. Furthermore, our data suggest that the fiberassociated Lachnospiraceae-type microbiome has a protective role in the orthotopic model and induces a systemic $T$ cell response, suggesting its contribution to a tumor-inhibiting microenvironment. These findings emphasize the important effects that microbiota abundance and composition can exert on immunomodulatory signals derived from the tumor microenvironment. This study, in accordance with investigations of CRC patients, supports the existence of a cancerspecific signature ${ }^{5,35}$. These putative "oncobacteria" include Clostridium XIVa pathobionts ( $C$. symbiosum, C. asparagiforme, C. hylemonae, C. bolteae, and C. hathewayl $)^{39}$, Bacteroides sp., including $B$. fragilis ${ }^{40,41}$, as well as the high-sulfur-metabolizing $D$. piger ${ }^{42}$. In addition, previous studies in mice suggested that microbiota composition and structural organization contribute to CRC oncogenesis $^{43,44}$. However, many of the specific microbiota members associated with such responses vary substantially between studies ${ }^{45}$. For instance, none of the donors used in our study had detectable levels of Fusobacterium nucleatum, a known pro-carcinogenic bacterium that has been correlated with clinical outcome ${ }^{8,46}$. However, this is also consistent with studies where the presence of $F$. nucleatum in CRC biopsies largely varies between individuals ${ }^{4}$.

It is not currently clear whether the altered microbiome in CRC patients contributes to or is a consequence of disease. It may therefore be relevant that the pre-tumor (week 2) microbiota was predictive of tumor growth, which suggests that the pre-cancer microbiome may establish a permissive or protective environment for CRC development. Notably, here we identified a dominant human Lachnospiraceae microbiota as being protective in the mouse model. Specifically, 
commensals including $C$. comes, $R$. lactaris, B. longum, and $B$. pseudocatenulatum were largely responsible for this effect. Bifidobacterium spp. are known for their immunomodulatory effects ${ }^{47}$ and they were found to be depleted in CRC patients ${ }^{32,48}$, as well as in biofilm positive-inoculated mice ${ }^{44}$.

Of note, the commensal $B$. longum has been reported to be enriched in patients with metastatic

melanoma that respond to PD-1 immunotherapy and associated with improved immune-mediated tumor control ${ }^{49}$. This "protective" microbiome concept is consistent with the fact that the microbiome from mice that received the Lachnospiraceae CAG harbored a significantly higher abundance of health-related taxa groups ${ }^{39}$ (i.e. lost in disease), in the elderly and across multiple diseases, compared to the microbiome of mice that received the Pathogen CAG (Fig. S18).

The occurrence of genetic alterations involved in the initiation of human CRC has been suggested to be increased by a "dysbiotic" microbiota ${ }^{6}$. In that context, we suggest that it is relevant for CRC progression that the CAG microbiome types tested here contributed differently to the cancer hallmarks, including changes in the expression levels of genes involved in epithelial-mesenchymal transition, ${ }^{50,51}$, angiogenesis, and immune evasion. These pathways, which are associated with poor prognosis in $\mathrm{CRC}^{50}$, were overexpressed in patients harboring the Pathogen CAG. Interestingly, a recent study using a novel cancer mouse model with aberrant expression of the Zeb2 gene, a master regulator of the epithelial-mesenchymal transition, showed that these mice were characterized by microbial dysbiosis and that the microbiota was necessary for the development of CRC in Zeb2overexpressing mice ${ }^{51}$. It will now be interesting to investigate if the specific microbial components associate with consensus molecular subtypes ${ }^{52}$.

The composition and functionality of the immune infiltrate of the cancer patient are relevant for disease progression, metastasis, and immunotherapy treatment ${ }^{53}$. Abnormal immune responses, which are often accompanied by the recruitment of pro-inflammatory myeloid cells and overproduction of inflammatory cytokines, are associated with changes in the gut microbiota ${ }^{6}$. Specific bacterial species (e.g. B. fragilis and $P$. anaerobius) have been shown to trigger the secretion of chemokines that recruit immunosuppressive MDSCs, tumor-associated macrophages, and tumor-associated neutrophils ${ }^{54,55}$. Albeit working in a pre-clinical model, we identified differences in the numbers of immune cells in the spleens of mice harboring the two different microbiota types, which is consistent 
354 with the transcriptomic differences observed in the tumor-immune genes of this cohort of CRC 355 patients. Manipulation of the microbiota with the goal to modulate towards a "cold" tumor, 356 characteristic of patients with sporadic CRC, towards a "hot" tumor environment, which is 357 characterized by a high infiltration of T cells and an effective response to checkpoint blockade, is a 358 promising and valid therapeutic avenue, especially when in combination with current 359 immunotherapies.

Although we compared our findings in the mouse model to six large human CRC studies and confirmed many of the identified key taxa, we acknowledge the limitation of the use of only one representative donor for each microbiota type. Another limitation in this study is the fact that it is not powered to detect very small differences in human genes between the two different CAGs due to the small number of patient samples analyzed, which limits our ability to detect interactions between specific driver mutations and microbiome structure. Moreover, whether the microbiota-induced immunity affects the mutation burden of the tumor or vice-versa, remains to be determined.

In this study, we expanded on previous work by us and others to identify specific taxa and respective metabolites that correlate with $\mathrm{CRC}$ development and to suggest a mechanism that involves immune modulation by the microbiota and its metabolites. Future studies involving the culture of these microbial taxa, comparative genomics to evaluate strain-specificity and variability, and in vitro functional assays with defined bacterial consortia and/or their metabolites will be essential to understand if (and which) bacterial compounds can ultimately be used as immunomodulators in CRC. 
References

1. Bray, F. et al. Global cancer statistics 2018: GLOBOCAN estimates of incidence and mortality worldwide for 36 cancers in 185 countries. Ca Cancer J Clin 68, 394-424 (2018).

377 2. O'Keefe, S. J. D. Diet, microorganisms and their metabolites, and colon cancer. Nat Rev 378 Gastroentero 13, 691-706 (2016).

379 3. Keum, N. \& Giovannucci, E. Global burden of colorectal cancer: emerging trends, risk factors and 380 prevention strategies. Nat Rev Gastroentero 16, 713-732 (2019).

4. Flemer, B. et al. Tumour-associated and non-tumour-associated microbiota in colorectal cancer. Gut 66, 633-643 (2017).

5. Wirbel, J. et al. Meta-analysis of fecal metagenomes reveals global microbial signatures that are specific for colorectal cancer. Nat Med 25, 679-689 (2019).

6. Janney, A., Powrie, F. \& Mann, E. H. Host-microbiota maladaptation in colorectal cancer. Nature 585, 509-517 (2020).

7. Helmink, B. A., Khan, M. A. W., Hermann, A., Gopalakrishnan, V. \& Wargo, J. A. The microbiome, cancer, and cancer therapy. Nat Med 25, 377-388 (2019).

8. Kostic, A. D. et al. Fusobacterium nucleatum Potentiates Intestinal Tumorigenesis and Modulates the Tumor-Immune Microenvironment. Cell Host Microbe 14, 207-215 (2013).

9. Brennan, C. A. \& Garrett, W. S. Fusobacterium nucleatum - symbiont, opportunist and oncobacterium. Nat Rev Microbiol 17, 156-166 (2019).

10. Arthur, J. C. et al. Intestinal inflammation targets cancer-inducing activity of the microbiota. Sci New York N Y 338, 120-3 (2012).

11. Pleguezuelos-Manzano, C. et al. Mutational signature in colorectal cancer caused by genotoxic pks + E. coli. Nature 11, 1-5 (2020).

12. Wu, S. et al. A human colonic commensal promotes colon tumorigenesis via activation of $\mathrm{T}$ helper type 17 T cell responses. Nat Med 15, 1016-1022 (2009).

13. Boleij, A. \& Tjalsma, $\mathrm{H}$. The itinerary of Streptococcus gallolyticus infection in patients with colonic malignant disease. Lancet Infect Dis 13, 719-724 (2013).

14. Tsoi, H. et al. Peptostreptococcus anaerobius Induces Intracellular Cholesterol Biosynthesis in Colon Cells to Induce Proliferation and Causes Dysplasia in Mice. Gastroenterology 152, 14191433.e5 (2017). 328 (2014). 
16. Rooks, M. G. \& Garrett, W. S. Gut microbiota, metabolites and host immunity. Nature reviews. Immunology 16, 341-352 (2016).

17. Belkaid, Y. \& Hand, T. W. Role of the microbiota in immunity and inflammation. Cell 157, 121141 (2014).

18. Fridman, W. H., Pagès, F., Sautès-Fridman, C. \& Galon, J. The immune contexture in human tumours: impact on clinical outcome. Nat Rev Cancer 12, 298-306 (2012).

19. Galon, J. et al. Type, Density, and Location of Immune Cells Within Human Colorectal Tumors Predict Clinical Outcome. Science 313, 1960-1964 (2006).

20. Mariani, F., Sena, P. \& Roncucci, L. Inflammatory pathways in the early steps of colorectal cancer development. World J Gastroentero 20, 9716-9731 (2014).

21. Flemer, B. et al. The oral microbiota in colorectal cancer is distinctive and predictive. Gut 67, 1454-1463 (2018).

22. Claesson, M. J. et al. Gut microbiota composition correlates with diet and health in the elderly. Nature 488, 178-184 (2012).

23. Bindea, G. et al. Spatiotemporal Dynamics of Intratumoral Immune Cells Reveal the Immune Landscape in Human Cancer. Immunity 39, 782-795 (2013).

24. Li, X., Zhong, Q., Luo, D., Du, Q. \& Liu, W. The prognostic value of CXC subfamily ligands in

25. Chen, B., Khodadoust, M. S., Liu, C. L., Newman, A. M. \& Alizadeh, A. A. Cancer Systems Biology, Methods and Protocols. Methods Mol Biology Clifton N J 1711, 243-259 (2018).

26. Newman, A. M. et al. Determining cell type abundance and expression from bulk tissues with digital cytometry. Nat Biotechnol 37, 773-782 (2019).

27. Zigmond, E. et al. Utilization of murine colonoscopy for orthotopic implantation of colorectal cancer. PloS one 6, e28858 (2011).

28. Zitvogel, L., Pitt, J. M., Daillère, R., Smyth, M. J. \& Kroemer, G. Mouse models in oncoimmunology. Nature reviews. Cancer 16, 759-773 (2016).

29. Aidy, S. E. et al. Temporal and spatial interplay of microbiota and intestinal mucosa drive establishment of immune homeostasis in conventionalized mice. Mucosal Immunol 5, 567-579 434 (2012).

30. Donigan, M. et al. Novel murine model for colon cancer: non-operative trans-anal rectal injection. The Journal of surgical research 154, 299-303 (2009). 
32. Feng, Q. et al. Gut microbiome development along the colorectal adenoma-carcinoma sequence. Nature communications 6, 6528 (2015).

33. Vogtmann, E. et al. Colorectal Cancer and the Human Gut Microbiome: Reproducibility with Whole-Genome Shotgun Sequencing. PloS one 11, e0155362 (2016).

34. Zeller, G. et al. Potential of fecal microbiota for early-stage detection of colorectal cancer. Mol Syst Biol 10, 766 (2014).

35. Thomas, A. M. et al. Metagenomic analysis of colorectal cancer datasets identifies cross-cohort microbial diagnostic signatures and a link with choline degradation. Nat Med 25, 667-678 (2019).

36. Yu, J. et al. Metagenomic analysis of faecal microbiome as a tool towards targeted non-invasive biomarkers for colorectal cancer. Gut 66, 70-78 (2017).

37. Hannigan, G. D., Duhaime, M. B., Ruffin, M. T., Koumpouras, C. C. \& Schloss, P. D. Diagnostic Potential and Interactive Dynamics of the Colorectal Cancer Virome. Mbio 9, e02248-18 (2018).

38. Zhang, X.-H., Ma, J., Smith-Warner, S. A., Lee, J. E. \& Giovannucci, E. Vitamin B6 and colorectal cancer: Current evidence and future directions. World J Gastroentero 19, 1005-1010 (2013).

39. Ghosh, T. S., Das, M., Jeffery, I. B. \& O'Toole, P. W. Adjusting for age improves identification of gut microbiome alterations in multiple diseases. eLife 9, 211 (2020).

40. Goodwin, A. C. et al. Polyamine catabolism contributes to enterotoxigenic Bacteroides fragilisinduced colon tumorigenesis. Proceedings of the National Academy of Sciences 108, 15354-15359 (2011).

41. Chung, L. et al. Bacteroides fragilis Toxin Coordinates a Pro-carcinogenic Inflammatory Cascade via Targeting of Colonic Epithelial Cells. Cell Host Microbe 23, 421 (2018).

42. Nguyen, L. H. et al. Association Between Sulfur-Metabolizing Bacterial Communities in Stool and Risk of Distal Colorectal Cancer in Men. Gastroenterology 158, 1313-1325 (2020).

43. Baxter, N. T., Zackular, J. P., Chen, G. Y. \& Schloss, P. D. Structure of the gut microbiome following colonization with human feces determines colonic tumor burden. Microbiome 2, 20 (2014).

44. Tomkovich, S. et al. Human colon mucosal biofilms from healthy or colon cancer hosts are carcinogenic. J Clin Investigation 130, 1699-1712 (2019).

45. Borges-Canha, M., Portela-Cidade, J. P., Dinis-Ribeiro, M., Leite-Moreira, A. F. \& PimentelNunes, P. Role of colonic microbiota in colorectal carcinogenesis: A systematic review. Revista Española De Enfermedades Dig 107, 659-71 (2015).

46. Bullman, S. et al. Analysis of Fusobacterium persistence and antibiotic response in colorectal cancer. Science 358, eaal5240 (2017). 
47. O'Neill, I., Schofield, Z. \& Hall, L. J. Exploring the role of the microbiota member Bifidobacterium in modulating immune-linked diseases. Emerg Top Life Sci 1, 333-349 (2017).

48. Chen, W., Liu, F., Ling, Z., Tong, X. \& Xiang, C. Human Intestinal Lumen and Mucosa-Associated Microbiota in Patients with Colorectal Cancer. Plos One 7, e39743 (2012).

49. Matson, V. et al. The commensal microbiome is associated with anti-PD-1 efficacy in metastatic melanoma patients. Science 359, 104-108 (2018).

50. Punt, C. J. A., Koopman, M. \& Vermeulen, L. From tumour heterogeneity to advances in precision treatment of colorectal cancer. Nat Rev Clin Oncol 14, 235-246 (2017).

51. Slowicka, K. et al. Zeb2 drives invasive and microbiota-dependent colon carcinoma. Nat Cancer 1, 620-634 (2020).

52. Guinney, J. et al. The consensus molecular subtypes of colorectal cancer. Nat Med 21, 13501356 (2015).

53. Grivennikov, S. I., Greten, F. R. \& Karin, M. Immunity, inflammation, and cancer. Cell 140, 88399 (2010).

54. Orberg, E. T. et al. The myeloid immune signature of enterotoxigenic Bacteroides fragilis-induced murine colon tumorigenesis. Mucosal Immunol 10, 421-433 (2017).

55. Long, X. et al. Peptostreptococcus anaerobius promotes colorectal carcinogenesis and modulates tumour immunity. Nat Microbiol 4, 2319-2330 (2019).

56. Yu, Z. \& Morrison, M. Improved extraction of PCR-quality community DNA from digesta and fecal samples. undefined 36, 808-812 (2004).

57. Tran, T. T. T. et al. Prebiotic supplementation in frail older people affects specific gut microbiota taxa but not global diversity. Microbiome 7, 39 (2019).

58. Ntemiri, A. et al. Retention of Microbiota Diversity by Lactose-Free Milk in a Mouse Model of Elderly Gut Microbiota. J Agr Food Chem 67, 2098-2112 (2019).

59. Klindworth, A. et al. Evaluation of general 16S ribosomal RNA gene PCR primers for classical and next-generation sequencing-based diversity studies. Nucleic acids research 41, e1 (2013).

60. Bolger, A. M., Lohse, M. \& Usadel, B. Trimmomatic: a flexible trimmer for Illumina sequence data. Bioinformatics 30, 2114-2120 (2014).

61. Magoč, T. \& Salzberg, S. L. FLASH: fast length adjustment of short reads to improve genome assemblies. Bioinformatics (Oxford, England) 27, 2957-2963 (2011).

62. Caporaso, J. G. et al. QIIME allows analysis of high-throughput community sequencing data. Nature methods 7, 335-336 (2010). 
503 63. Edgar, R. C. Search and clustering orders of magnitude faster than BLAST. Bioinformatics 504 (Oxford, England) 26, 2460-2461 (2010).

505

64. Edgar, R. C., Haas, B. J., Clemente, J. C., Quince, C. \& Knight, R. UCHIME improves sensitivity and speed of chimera detection. Bioinformatics 27, 2194-2200 (2011).

65. Schloss, P. D. et al. Introducing mothur: open-source, platform-independent, communitysupported software for describing and comparing microbial communities. Applied and environmental microbiology 75, 7537-7541 (2009).

66. Allard, G., Ryan, F. J., Jeffery, I. B. \& Claesson, M. J. SPINGO: a rapid species-classifier for microbial amplicon sequences. Bmc Bioinformatics 16, 324 (2015).

67. Caporaso, J. G. et al. PyNAST: a flexible tool for aligning sequences to a template alignment. Bioinform Oxf Engl 26, 266-7 (2009).

68. Keohane, D. M. et al. Microbiome and health implications for ethnic minorities after enforced lifestyle changes. Nat Med 1-7 (2020) doi:10.1038/s41591-020-0963-8.

69. Langmead, B. \& Salzberg, S. L. Fast gapped-read alignment with Bowtie 2. Nat Methods 9, 357359 (2012).

70. Truong, D. T. et al. MetaPhIAn2 for enhanced metagenomic taxonomic profiling. Nat Methods 12, 902-903 (2015).

71. Franzosa, E. A. et al. Species-level functional profiling of metagenomes and metatranscriptomes. Nat Methods 15, 962-968 (2018).

72. Truong, D. T., Tett, A., Pasolli, E., Huttenhower, C. \& Segata, N. Microbial strain-level population structure and genetic diversity from metagenomes. Genome Res 27, 626-638 (2017).

73. Pasolli, E. et al. Accessible, curated metagenomic data through ExperimentHub. Nat Methods 14, 1023-1024 (2017).

74. Fein, M. R. et al. Cancer cell CCR2 orchestrates suppression of the adaptive immune response. J Exp Medicine 217, (2020). 
530 Acknowledgments: We thank the APC Germ-Free facility staff (Frances O'Brien and Tara

531 O'Driscoll), APC Flow Cytometry manager Pepi Stamou; APC research nurses (Orlagh O'Connor, 532 Joanne Carroll, and Ciara Tobin); Juliet Barry at Cork Cancer Research Centre (CCRC) for 533 processing histology samples; and Jan Soetaert (QMUL) for assistance on image analysis. Special 534 thanks to all patients that generously participated in this study. The graphical abstract and figures $1 \mathrm{~A}$ 535 and 2B were created with Biorender.com

537 Funding: This project received funding from the European Union Horizon 2020 research and 538 innovation program under the Marie Skłodowska-Curie grant agreement No 752047 to ASA. ASA and 539 LAA were both recipients of a PARSUK-Xperience 2017 scholarship funded by Fundacao Calouste 540 Gulbenkian. IS and RK were funded by a Barts Charity (MGU045). This work was supported, in part, 541 by Science Foundation Ireland through a Centre award to APC Microbiome Ireland (12/RC/2273_P2).

Conflict of Interests: None

Author contributions: ASA, FS and PWOT designed the study; ASA, TTT, TSG, CR, and BF performed microbiota data analysis; CF performed RNAseq analysis on human samples; ASA, LAA, $\mathrm{PP}, \mathrm{WF}, \mathrm{CMH}$ and RJ performed experiments; ASA, RJ and IS performed immune infiltrate data analysis; MOR was responsible for patient sample acquisition; ASA and PWOT wrote the manuscript and obtained funding for the study. 


\section{$551 \quad$ Figure Legends}

552 Figure 1. Distinct microbiomes in patients with adenomas and CRC correlate with differential 553 human immune transcripts

554 A. Overview of the experimental design. 32 treatment-naïve patients were included in the study. 555 Surgical resections were collected from multiple sites in the colon and analyzed by $16 \mathrm{~S}$ rRNA sequencing, immunofluorescence, and RNA expression analysis. Selected fecal samples were collected anaerobically and administered in a germ-free cancer mouse model. B. Human microbiota composition measured by proportional abundance of bacterial CAGs in human colon biopsies. CAGs are named after the most abundant genus. Stars $\left(^{*}\right)$ indicate the 12 patients selected for bulk RNAseq analysis. C. Venn diagram depicting numbers of significantly DEGs $(p$ value $<0.5)$ between healthy controls (Healthy, $n=10$ ), tumors from the Pathogen CAG (Path, $n=6$ ), and tumors from subjects harboring the Lachnospiraceae CAG (Lachno, $\mathrm{n}=6$ ). The gene numbers circled in red are those uniquely elevated in the Pathogen CAG and gene numbers circled in green are uniquely elevated in the Lachnospiraceae CAG. D. Heatmap of unsupervised hierarchal clustering of genes and patients, representing the top 60 significantly DEGs (CRC versus healthy controls; FDR adjusted $p$ value $<0.1$, and Lachnospiraceae CAG versus Pathogen CAG; $p$ value $<0.05$. All $\log _{2} \mathrm{FC} \leq-1.5$ and $\geq 1.5$ ), consisting of the top 10 significantly DEGs from each circle highlighted in the Venn diagram (panel

C). E, F. Expression plots displaying labeled immune genes differentially expressed and uniquely elevated in (E) Pathogen CAG and (F) Lachnospiraceae CAG tumors relative to healthy controls. The $\mathrm{x}$-axis is the logCPM values for healthy controls and the $y$-axis is the logCPM values for (E) Pathogen CAG and (F) Lachnospiraceae CAG tumors. Red dots show genes of interest. G, H. Estimated immune cell abundancies from whole transcriptomic data deconvoluted with the CIBERSORTx software in healthy controls and tumors. Estimated lymphocyte abundances were calculated as the sum of proportions of naïve B cells, memory B cells, CD8 ${ }^{+} T$ cells, naïve CD4 ${ }^{+} T$ cells, resting memory $\mathrm{CD}^{+} \mathrm{T}$ cells, and activated memory $\mathrm{CD} 4^{+} \mathrm{T}$ cells. Estimated monocyte abundances were calculated as the sum of proportions of monocytes, M0 macrophages, M1 macrophages, and M2 macrophages. $0.01,{ }^{* * *} p \leq 0.001,{ }^{* * * *} p \leq 0.0001$. 

model of CRC.

A. Validation of microbiota composition in human donors for the pre-clinical mouse trial. Colon resections were collected at surgery and the mucosal microbiota was profiled using 16S rRNA sequencing. Pie-charts represent the abundance of the five bacterial CAGs on adenoma or tumor samples from each donor. Donor 1 (CRC044), diagnosed with a T3N2 rectum adenocarcinoma, was selected based on the high abundance of a Pathogen CAG microbiota and Donor 2 (CRC056), diagnosed with a tubulovillous adenoma, was selected based on the high abundance of a Lachnospiraceae CAG microbiota. B. Experimental design of the pre-clinical trial with a humanized

C. Tumor growth is reduced in mice receiving the Lachnospiraceae microbiota compared to mice receiving the Pathogen CAG microbiota or control germ-free (GF) mice. Tumor volume was measured with a caliper at endpoint and calculated as (length $\mathrm{x}$ width $\left.{ }^{2}\right) / 2$. Overall $p$ values were calculated with the Kruskal-Wallis test. Data indicate mean \pm SEM. $n=3-6$ replicates/group per condition. Data from two independent experiments are shown by open and grey bars. D. Relatedness ( $\beta$-diversity) of the fecal microbiota of the two human donors and respective recipient mice at different time-points represented by principal coordinate analysis (PCoA) on Bray-Curtis distance matrix (PERMANOVA $r^{2}=0.79 ; p$ value $\left.=0.001\right) . \mathrm{W}$, week. E PCoA plots of the Metaphlan2 species-level taxa profiles of the murine fecal microbiomes, performed at week 2 (left) and week 5 (right). Fecal microbiome profiles corresponding to the Pathogen CAG donor and the Lachnospiraceae CAG donor are colored in red and green, respectively. Each point corresponds to a specific mouse ID and the corresponding tumor volume is shown within parentheses. The size of each point is proportional to the tumor volume. PERMANOVA $\mathrm{r}^{2}=0.24 ; p=0.016$ (week 2$), \mathrm{r}^{2}=0.25 ; p$ value $=0.006$ (week 5).

\section{Figure 3. Distinct bacterial taxa are associated with final tumor volume}

Heatmap showing the ranked abundances of 'tumor positive' (red) and 'tumor negative' (green) taxa in mice fecal microbiomes at week 2 only (top panel), shared between week 2 and 5 (middle panel), and week 5 only (bottom panel), as determined by shallow shotgun sequencing of samples from experiment 2. For each mouse, the corresponding tumor volume at week 5 is indicated as bar plots 
607 at the top of the heatmap. Spearman's rank correlations between bacterial taxa abundance at different

608 time points and the tumor volume at week 5 are indicated. The effect sizes (Cohen's D) observed for 609 the various taxa in the Global Reference cohort are also shown. Taxa that are significantly enriched 610 or depleted in CRC $(n=325)$ versus healthy individuals $(n=310)$ (identified using Mann-Whitney $U$ 611 tests, $p$ values corrected using Benjamini-Hochberg for FDR $<0.1$ ) are indicated by red and green 612 stars, respectively. Positive association with tumor volume and enrichment in CRC in the Global 613 Reference cohort is color-coded in red. Negative association with tumor volume and depletion in CRC 614 in the Global Reference cohort is color-coded in green.

Figure 4. High-risk and low-risk microbiome are associated with different metabolic pathways

A. Pre-tumor microbiota is predictive of tumor growth. Tumor-associated bacterial taxa at week 2 have higher predictability for tumor volume than taxa at week 5 . Boxplots show the variation of Spearman rho values calculated between the predicted and actual tumor volumes obtained for the 100 iterations of the two variants of RF models (trained on week 2 and week 5 abundance profiles, respectively). Mann-Whitney $U$ test $p$ values for the different comparisons are indicated. B. Volcano plot showing the validated set (identified as summarized in Fig. S13) of metabolite production functionalities that were predicted to have either a significant positive or negative association with the Pathogen CAG microbiome. The $x$-axis indicates the effect size difference (negative indicating enriched in the Lachnospiraceae CAG and positive indicating enriched in the Pathogen CAG), and the $y$-axis indicates the negative log of FDR value. C. Boxplots comparing (left) the coverage of the bile acid inducible (bai) gene cluster that converts the primary bile acids (cholic acid and chenodeoxycholic acid) into secondary bile acids (deoxycholic acid and lithocholic acid); (middle) the cumulated gene abundances of $\mathrm{CntA}$ and CutA enzymes that catalyze trimethylamine (TMA) production; and, (right) the abundance of the AtoD enzyme catalyzing the last step of short-chain fatty acids formation, between the Pathogen and the Lachnospiraceae microbiome types. The $p$ values obtained using the Mann-Whitney $U$ tests are indicated. 
634 Figure 5. Lachnospiraceae-type microbiome colonization induces a strong immune infiltration 635 and antitumoral immune response.

636 A. Spleens from mice with the Pathogen CAG have more neutrophils $\left(\mathrm{CD} 45^{+} \mathrm{CD} 11 \mathrm{~b}^{+} \mathrm{MHCll}\right.$

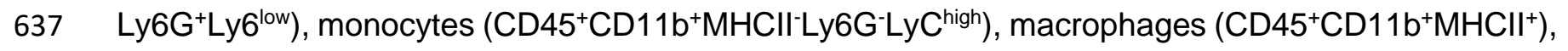
638 and dendritic cells $\left(\mathrm{CD} 45^{+} \mathrm{MHCI}{ }^{+} \mathrm{CD} 11 \mathrm{~b}-\mathrm{CD} 11 \mathrm{c}^{+}\right)$, as determined by flow cytometry gated on $\mathrm{CD}^{+} 5^{+}$ 639 cells. Panels show quantification of neutrophils, monocytes, macrophages, and dendritic cells. B. 640 Spleens from mice with the Lachnospiraceae CAG have more $\mathrm{CD}^{+}, \mathrm{CD}^{+}, \mathrm{CD}^{+}$, and NK T cells, as 641 determined by flow cytometry gated on CD45+ cells. $P$ values were determined by Mann-Whitney U 642 test and are represented in each plot. Data indicate mean \pm SEM. $n=6$ biological replicates/group. C. 643 Quantification of immune infiltrate $\left(C D 45^{+}\right)$, T-cell infiltrate $\left(C D 3^{+}\right.$and $\left.C D 8^{+}\right)$and neutrophil infiltrate $644\left(C D 15^{+}\right)$in human CRC biopsies from Pathogen $(n=5)$ and Lachnospiraceae-enriched tumors $(n=4)$. 6452 sections/tumor and 3 ROls quantified per section (same ROls were used to quantify different 646 immune cell subpopulation in each tumor), means are shown, group comparison with one-way 647 ANOVA. D. Immunofluorescence representative images of Pathogen CAG-enriched tumors 648 (CRC073) and Lachnospiraceae CAG-enriched tumors (CRC057) human tumors showing that more $649 \mathrm{CD}^{+} \mathrm{T}$ cells (red) infiltrate into Lachnospiraceae tumors, while more CD15+ neutrophils infiltrate into 650 Pathogen tumors. Counterstained with nuclear dye DAPI. All tumors were analyzed and for each 651 tumor; 3 ROls were quantified per section ( $n=2$ sections/tumor/staining). Scale bars $100 \mu m$. 


\section{Materials and Methods}

\section{Patients cohort and sample collection}

654 All clinical studies were conducted after informed consent of the patients, following the guidelines of 655 the Declaration of Helsinki. Ethical approval for this study was granted by the Clinical Research Ethics 656 Committee of the University College Cork, under the study number APC033. Patients' data were anonymized and stored under European Union General Data Protection Regulation. Detailed clinical and pathological information on the patients is presented in Table S1. A total of 32 treatment-naïve patients with adenomas or CRC were included in this study. Exclusion criteria included a personal history of CRC, inflammatory bowel disease (IBD), or inflammatory bowel syndrome (IBS), as well as chemotherapy or radiation therapy treatments. Individuals were not treated with antibiotics in the month prior to surgery but were administered antibiotics intravenously during surgery. Dietary data for each patient were collected using a validated Food Frequency Questionnaire $(F F Q)^{22}$. Control subjects (i.e. routine colonoscopy) included individuals without a history of CRC, IBD or IBS, or antibiotic usage within 3 months, described elsewhere ${ }^{4}$.

Resection samples were collected from CRC patients undergoing surgery at Mercy University

Hospital, Cork. Samples were collected from the tumor site $(\mathrm{ON}), 2-3 \mathrm{~cm}$ far from the tumor margin (OFF), and paired healthy tissue (approximately $10 \mathrm{~cm}$ from the tumor site). Samples collected were

rapidly preserved in 1) RNA later for sequencing purposes; 2) methacarn for histology and 3) snap-

frozen for subsequent analysis. Bowel preparations before surgery or colonoscopy were determined by the surgeon and are detailed in Table S1.

DNA and RNA extraction of human biopsies

674 Human colon resections were placed in RNAlater (Qiagen) at the time of resection, stored at $4^{\circ} \mathrm{C}$ for $12 \mathrm{~h}$, and then stored at $-20^{\circ} \mathrm{C}$ until processing. Genomic DNA and total RNA were extracted using the AllPrep DNA/RNA kit (Qiagen). For tissue samples, 20 mg of tissue was placed into bead tubes containing $250 \mu \mathrm{l}$ of $0.1 \mathrm{~mm}$ sterile glass beads (Biospec Products) and three $3-4 \mathrm{~mm}$ sterile glass beads (Biospec Products). Next, $600 \mathrm{~mL}$ of buffer RLT (Qiagen) containing 1\% $\beta$-mercaptoethanol 
at full speed. The extraction was then carried out using the AllPrep DNA/RNA extraction kit (Qiagen), following the manufacturer's instructions. Genomic DNA was quantified using the Nanodrop 1000 (Thermo Scientific) and total RNA was quantified using the Bioanalyser (Agilent).

\section{Human fecal inoculum preparation}

Two human donors were selected from the CRC patients' cohort based on their mucosal microbiota compositional profiles. Donor 1 (CRC044) is a female, 66 years old, diagnosed with a T3N2b rectum adenocarcinoma; donor 2 (CRC056) is a male, 65 years old, diagnosed with a tubulovillous adenoma with low-grade dysplasia (Table 1 and Figure 2A). Stool samples were transferred to an anaerobic chamber immediately after voiding, transported to the lab in anaerobic bags, and transferred to an anaerobic hood in less than an hour. Fresh stools were diluted (ratio 1:10) in sterile pre-reduced PBS with $20 \%$ glycerol and stored at $-80^{\circ} \mathrm{C}$ in aliquots until further use.

\section{DNA extraction of human fecal samples}

Genomic DNA was extracted from fecal samples following the Repeated Beat Beating (RBB) Method ${ }^{56}$, with the following modifications. Samples $(0.25 \mathrm{~g})$ were placed in sterile tubes containing one $3.5 \mathrm{~mm}$ zirconia bead and one scoop of $0.1 \mathrm{~mm}$ and $1 \mathrm{~mm}$ beads, respectively (Thistle Scientific, UK). Fecal samples were homogenized via bead beating for 60 seconds (Mini-Beadbeater ${ }^{\mathrm{TM}}$, BioSpec Products), with the samples cooled on ice for 60 seconds before another 60 seconds bead beating. Samples were then incubated at $70^{\circ} \mathrm{C}$ for $15 \mathrm{~min}$ and centrifuged at full speed for $5 \mathrm{~min}$. Pooled supernatants were incubated with $350 \mathrm{ml}$ of $7.5 \mathrm{M}$ ammonium acetate (Sigma) and incubate on ice for $5 \mathrm{~min}$. The remaining steps of DNA purification were performed using QIAamp columns (Qiagen). Genomic DNA was quantified using the Nanodrop 1000 (Thermo Scientific). Extracted genomic DNA was stored at $-20^{\circ} \mathrm{C}$ until amplification.

\section{MC-38 cells culture}

Murine C57BL/6 MC-38 colorectal tumor cells were obtained from Kerafast (ENH204-FP) and maintained in $5 \% \mathrm{CO}_{2}$ at $37^{\circ} \mathrm{C}$ in Dulbecco's modified MEM (DMEM) medium supplemented with $10 \%$ 
heat-inactivated fetal bovine serum (Sigma), 2mM L-glutamine, $0.1 \mathrm{mM}$ nonessential amino acids, 1 mM sodium pyruvate, 10mM HEPES and 100 units/ml penicillin/streptomycin antibiotic solution (all reagents from Gibco-Invitrogen). Cells were tested for Mycoplasma contamination every 4 - 6 weeks and before each experiment (Mycoalert Mycoplasma Detection kit, Lonza).

\section{Germ-free MC-38 mouse model}

All animal protocols were approved by the Animal Experimentation Ethics Committee at University College Cork and by the Health Products Regulatory Authority (HPRA) of Ireland, per EU Directive 2010/63/EU (HPRA Project authorization number AE19130/P055).

Germ-free (GF) mice were bred and maintained at the APC Germ-Free facility in dedicated axenic isolators (Bell Isolation Systems). Germ-free status was routinely monitored by culture-dependent methods. Age-matched male C57BL/6 GF mice, 6-10 weeks of age, were group-housed 3-4 and transferred into sterile individual ventilated cages (IVCs) (Arrowmight, Hereford, UK) before undergoing human microbiota administration. Mice were kept in a 12-hour light-dark cycle and on ad libitum diet RM1 (autoclaved) (Special Diet Services, \#0103). Water and diet were batched at the beginning of the experiment to exclude possible variations between batches. An overview of the experimental study design is presented in Figure 2B. Animals were pipette-dosed with $100 \mu$ l fecal slurry or control PBS, per day for 3 consecutive days. Groups were as follows: "Pathogen CAG" group, inoculated with fecal slurry from donor 1 (patient CRC044); "Lachnospiraceae CAG" group, inoculated with fecal slurry from donor 2 (patient CRC056); and, GF control group inoculated with $100 \mu$ of reduced PBS + glycerol 20\%. After microbiota administration and colonization, MC-38 cells were orthotopically injected into the rectal submucosa, as previously described ${ }^{27}$. Briefly, mice were anesthetized using a mixture of Ketamine/Medetomidine (75 mg/kg ketamine (100mg/ml), $0.5 \mathrm{mg} / \mathrm{kg}$ medetomidine $(1 \mathrm{mg} / \mathrm{ml})$ subcutaneously. Injection of $20 \mu \mathrm{l}$ of $5 \times 10^{5}$ (experiment1) or $1 \times 10^{5}$ (experiment 2) MC-38 cancer cells was performed using an insulin syringe on the right flank. Fecal and blood samples were collected from each animal at various time points (weeks 0 to 5 ) as indicated in Figure 2B. Two to three fecal pellets were collected at each time point and were immediately frozen in dry ice before being transferred to $-80^{\circ} \mathrm{C}$. Tumor size was measured by caliper at endpoint (week 
4 in experiment 1 and week 5 in experiment 2), and tumor volume was calculated as (length $\times$ width $\left.^{2}\right) / 2$.

\section{Genomic DNA extraction and microbiota profiling of murine fecal samples}

Total DNA was extracted from mouse fecal samples using QIAamp DNeasy Blood and Tissue Kit (Qiagen, UK) according to the manufacturer's protocol and as previously described ${ }^{57}$. Genomic DNA was quantified using the Nanodrop 1000 (Thermo Scientific, Ireland). Extracted genomic DNA was stored at $-20^{\circ} \mathrm{C}$ until amplification.

\section{Sequencing, Taxonomic and Functional Profiling}

16S rRNA Gene Amplicon Sequencing: The V3-V4 region of the 16S rRNA gene was amplified, sequenced, and analyzed as described before ${ }^{58}$. Amplification was performed with the universal $16 \mathrm{~S}$ rRNA gene primer pair S-D-Bact-0341-b-S-17 and S-D-Bact-0785-a-A-2159. The Phusion HighFidelity PCR Master Mix (ThermoFisher Scientific, USA) was used for the amplification. The sequencing library was prepared using the Nextera XT V.2 Index Kit (Sets A and D, Illumina) according to the Illumina $16 \mathrm{~S}$ MiSeq Sequencing Library protocol. The PCR products were purified with the SPRIselect reagent kit (Beckman Coulter, Inc., USA). Amplicons were quantified with a Qubit dsDNA HS Assay Kit (Thermo Fischer Scientific) and pooled at the same concentration. Sequencing was performed on an Illumina MiSeq Platform (2x250 bp reads for human samples and 2×300 bp reads for mouse samples) by the Teagasc Next Generation DNA Sequencing Facility (Fermoy, Ireland).

Microbiota composition analysis of 16S rRNA amplicon sequencing data: Primers were removed from raw sequences using Trimmomatic ${ }^{60}$ (v0.36). Paired-end sequencing reads (2x250 bp or $2 \times 300 \mathrm{bp}$ ) were joined using FLASH ${ }^{61}$ (v1.2.8). Demultiplexing and quality filtering were performed using the QIIME package ${ }^{62}$ (v1.9.1). The USEARCH ${ }^{63}$ sequence analysis tool (v8.1.186) was applied for further quality filtering and de novo clustering to form operational taxonomic units (OTUs). The sequences were initially filtered by length and sorted by size, and single unique sequences were 
removed. The remaining sequences were clustered into OTUs at $97 \%$ similarity. Subsequently, chimeras were removed with $\mathrm{UCHIME}^{64}$, using the GOLD reference database. The original qualityfiltered sequences were mapped against the OTUs at $97 \%$ sequence identity. OTU representative sequences were classified with a confidence threshold of $80 \%$ to taxonomic ranks from phylum to genus level by mothur (v1.36.1) using the RDP reference database (trainset $16^{65}$ ) and to species level by SPINGO$^{66}(\mathrm{v} 1.3)$ using the RDP reference database (v11.4). Alpha $(\alpha)$ and beta $(\beta)$ diversity analyses were performed in QIIME on a rarefied OTU table. The sequences were aligned using the PyNast tool ${ }^{67}$ in Qllme to generate $\alpha$-diversity indices (Shannon, Simpson, PD whole tree, Chao1, and Observed Species), and $\beta$-diversity indices (Bray-Curtis, Weighted UniFrac, and Unweighted UniFrac).

\section{Shallow shotgun analysis}

Pre-processing of shotgun sequence data: Pre-processing of raw shotgun sequence reads was performed using a similar approach adopted by previous studies ${ }^{68}$. To summarize, the reads were quality trimmed using Trimmomatic (v0.39, with default parameters) ${ }^{60}$; followed by removing reads originating from the host genome bowtie2 (v2.3.4 with default parameters) (with Mus musculus genome version 9 for mouse fecal metagenomes and human genome hg38 for donor fecal metagenomes $)^{69}$.

Taxonomic, functional, and strain-wise profiling: The taxonomic and functional profiling of the metagenomes was performed using the HUMAnN2 pipeline with the clade-specific marker genebased metaphlan2 as the taxonomic classifier ${ }^{70,71}$. Pathway and gene-family abundances obtained using the UniProt mapping scheme were subsequently converted into the KEGG-specific mapping scheme using the legacy databases of humann2 (as described in Keohane et al. ${ }^{68}$ ). Strain-wise variations were profiled using Strainphlan $2^{72}$. Inferred metabolite production and consumption profiles were obtained using a similar approach as adopted in Ghosh et al. ${ }^{39}$. 


\section{RNA-Sequencing}

RNA sequencing libraries were prepared by Genewiz with the Standard RNA-seq protocol for tumor samples and by GATC for healthy control samples. Tumor samples were sequenced on an Illumina HiSeq instrument with 150-bp paired-end reads to an average depth of 29 million pairs of reads per sample. Healthy control samples were sequenced with 100-bp paired-end reads to an average depth of 45 million pairs of reads per sample.

\section{RNAseq transcriptome analysis}

FastQC software (v0.11.5) was performed to assess the quality control checks of paired-end sequencing reads. The TrimGalore (v0.6.5) tool was used with Cutadapt (v1.15) and FastQC to apply quality and adapter trimming to FASTQ files. STAR (v2.7.3a) was used to align trimmed reads to the human genome (Homo sapiens high coverage assembly GRCh38 from the Genome Reference Consortium - GRCh38.p13) with the --quantMode GeneCounts option to output read counts per gene. The Bioconductor package EdgeR (v3.28.1) was applied in R (v3.6.3) to identify statistically significant differentially expressed genes between patient groups. Biological and technical variation was accounted for by the negative binomial distribution of RNAseq count data using a generalization of the Poisson distribution model. The filterByExpr function was applied to remove lowly expressed genes. The data was normalized across library sizes, between samples using the trimmed mean of M-values (TMM) normalization method. Tagwise dispersions were estimated for the normalized dataset. $\mathrm{P}$ values from multiple comparisons were corrected with the Benjamini-Hochberg method in EdgeR. For the comparisons between tumors and healthy controls, genes were considered significantly differentially expressed with an FDR adjusted $p$ value $<0.1$. For the comparisons between Pathogen and Lachnospiraceae enriched tumor genes were considered significantly differentially expressed with a $p$ value $<0.05$. Voom "variance modeling at the observational level" method within edgeR was used to output normalized read counts as LogCPM values. These were used to perform hierarchical clustering and to construct heatmaps in Gene Pattern's online server (v3.9.11), volcano plots in Gene Patterns Multiplot Studio (v1.5.2), to estimate the abundances of immune cell types in a mixed cell population with CIBERSORTx ${ }^{25,26}$ signature genes (LM22), and to perform Gene Set 
820 Enrichment Analysis (GSEA) (v4.1.0) with annotated HALLMARK genesets from the MSigDB

821 (Molecular Signatures Database) collections (v6.2). Venn diagrams were constructed using

822 InteractiVenn. Further statistical analysis of estimated abundances of immune cell types from

823 CIBERSORTx involved students t-tests between patient groups within GraphPad Prism (v6).

824 Summary statistics for the RNA Seq data analysis is given in Table S3 and S4.

825

826

827

828

829

\section{Statistical analysis}

Statistical analysis, data visualization and machine learning-based analyses were carried out in $\mathrm{R}$ statistical software package (v3.4.0). Principal Coordinate Analyses (PCoA) were performed using the dudi.pco function of the ade4 package (v1.7-15). Two-dimensional PCoA plots were created using the ggplot2 package (v2.2.1). Permutational multivariate analysis of variance (PERMANOVA) analyses to test for statistical difference in $\beta$-diversity, the gut microbiome profiles as well as the strain variations of the different species were performed using the adonis function from the vegan package (v2.5-6). Spearman Distances of the species abundances across samples and the species-specific strain-wise distances were provided as inputs to the adonis function. PERMANOVA analysis of the strain-wise variations for each species (obtained using Strainphlan) was performed in a time-point specific manner (separately for week 2 and week 5), after adjusting for the donor and abundance of the given species as confounders. Effect size calculations (Cohen's D) were performed using the effsize package (v0.8.1). We excluded from the analysis any bacterial taxa or metabolites that were detected in less than $50 \%$ of the samples of each group. Significant variations in a-diversity, taxa/gene relative abundance, metabolites abundance, and pathway coverages were assessed on median values of the technical replicates using the Mann-Whitney $U$ test for unpaired data or Wilcoxon signedrank test for paired data. The Kruskal-Wallis test followed by Dunn's post hoc test with BenjaminiHochberg $p$ value adjustment for multiple testing was applied when comparing more than two experimental groups. The bar plots showing different taxonomic level classification were created using the ggplot 2 package. Taxa below $1 \%$ sample abundance and the unclassified taxa were grouped into the "Other" category. $P$ values from multiple comparisons were adjusted for the FDR using the Benjamini-Hochberg method (implemented in the p.adjust $R$ function). Significance was 


\section{Flow cytometry}

874 Spleens were harvested 19 days after MC-38 cancer cells injection (Experiment 2) and processed for 875 flow cytometry analysis as previously described ${ }^{74}$. For staining, single cells $\left(1 \times 10^{6}\right)$ were pre-

assumed for adjusted $p$ values $\leq 0.05$, if not stated otherwise. Correlations between metabolite and taxa relative abundances were calculated using standard Spearman's rank correlation using the 'corr' function implemented in the 'psych' module of R and hierarchical clustering was computed using the hclust function in R (method "complete"). Features (that is taxa at various time-points and inferred metabolites) that showed significant Spearman correlations with tumor volumes (FDR $<0.1$, obtained after $p$ value adjustment using the Benjamini-Hochberg method) were visualized using the ggplot2 package.

\section{Machine-learning based analysis}

For comparative validation on a global scale, we utilized the curatedMetagenomicData repository to six additional case-control datasets containing human fecal shotgun metagenome data from greater than 600 individuals consisting of CRC patients and controls ${ }^{73}$ (this was referred to as the 'Global Reference CRC cohort'32-37). For comparing taxa abundances or inferred metabolite inferences within this Global Reference CRC cohort, we adopted a two-step procedure. First, for inter-dataset variability in the detection of various taxa, we performed across sample rank normalizations of taxa abundances separately within each dataset corresponding to the six studies. This limited the abundance range of each taxon from 0 to 1 uniformly for all the six studies. Subsequently, the rank normalized profiles for the six studies were combined and the comparison of rank normalized taxa abundances were compared between CRC patients and non-diseased individuals using Mann-Whitney $U$ tests. Machine-learning based analyses consisting of evaluating the disease predictive ability of various markers in the Global Reference CRC cohort as well as within our dataset were performed using Random Forest approach (using the randomForest module implemented the R-programming interface). Iterative random forest classifiers built by taking repeated $50 \%$ subsets of 'test' and 'training' samples were obtained using the same methodology as used in Ghosh et al. ${ }^{39}$.

Fow cytometry 
incubated with purified anti-mouse CD16/CD32 (clone 2.4G2, Biolegend) for 10 min on ice, and then stained with the appropriate surface markers antibodies at $4^{\circ} \mathrm{C}$ for $30 \mathrm{~min}$ in the dark. Zombie Red (BioLegend) was used to differentiate between dead and live cells. The stained populations were analyzed using a BD FACSCelesta ${ }^{\mathrm{TM}}$ flow cytometer (BD, USA) and FlowJo software (BD, v10). Antibodies were titrated for optimal staining. Antibodies used were: CD45-BV510 (Clone 30-F11, dil 1/100), CD274 (B7-H1, PD-L1)-BV605 (Clone 10F.9G2, dil 1/100), Ly6G-BV786 (clone 1A8, dil 1/100), CD11b-FITC (clone M1/70, dil 1/100), CD103-PE (Clone 2E7, dil 1/200), F4/80-PerCP-Cy5.5 (Clone BM9, dil 1/80), CD11c-APC (Clone N418, dil 1/200), Ly6C-AF700 (clone HK1.4, dil 1/200), CD3-FITC (Clone 17A2, dil 1/100), NKp46-PE (clone 29A1.4, dil 1/50), CD4-PerCP-Cy5.5 (Clone RM4-4, dil 1/200), CD279 (PD-1)-APC (clone 29F.1A12, dil 1/200), CD8-AF700 (Clone 53-6.7, dil 1/200), all from BioLegend; MHC II (I-A/I-E)-APC-eFluor 780 (Clone M5/114.15.2, dil 1/200) from eBioscience.

\section{Immunofluorescence staining, imaging, and quantification}

Methacarn-fixed paraffin sections of human CRCs were blocked with $10 \%$ fetal bovine serum, $2 \%$ BSA, $0.02 \%$ fish skin gelatin, $0.05 \%$ TritonX100 (Sigma) and $0.05 \%$ Tween (Sigma). After $1 \mathrm{~h}$ blocking at room temperature, primary antibodies were incubated overnight at $4^{\circ} \mathrm{C}$, followed by $2 \mathrm{~h}$ incubation at room temperature in secondary antibody.

The following primary antibodies were used: mouse anti-CD45 (Leica Biosystems, NCL-L-LCA, 1/70), mouse anti-CD3 (Leica Biosystems CD3-565-L-CE, 1/100), mouse anti-CD8 (Novus Biologicals, NBP2-32952, 1/200), mouse anti-CD15 (Cell Signalling, SSEA1 MC480 \#4744S, 1/1500). AlexaFluor555 donkey anti-mouse (all Invitrogen, purchased from ThermoFisher Scientific, 1/300) as use used as secondary antibody. DAPI (Life Technologies) was used as a nuclear counterstain. Slides were mounted using ProLong Gold anti-fade reagent (Life Technologies). TissueFAXS Quantitative Imaging System (TissueGnostics, Vienna, Austria) was used to acquire images from the slides. The TissueFAXS uses a standard widefield epi-fluorescence Zeiss AXIO Observer.Z1 Inverted Microscope (with high efficiency fluorochrome specific DAPI, GFP, CFP, Cy3, and Cy5). Images were captured with a Hamamatsu ORCA-Flash 4.0 CMOS Camera. The entire slide $\left(75 \times 25 \mathrm{~mm}^{2}\right)$ was 
scanned at low magnification using a $5 \times$ objective to identify the location of the tissue on the slide,

followed by acquisition in multiple sequential tiles at $20 \times$ high magnification used for all downstream analysis. Image processing and analysis was performed using StrataQuest software version

6.0.1.209 (TissueGnostics, Vienna, Austria). Image processing included reconstruction of whole images and creation of in silico multiplexed images. Tissue cytometry and backgating into the tissue images were used for quantitation and visualization of the in silico data. In brief, several algorithms were used: to isolate cells by DAPI staining, to create a ring mask and identify non-nuclear staining starting from the centroid of the identified nucleus and stopping at the exterior of the biomarker, to identify the biomarker for the cell phenotype (CD45, CD3, CD8, CD15 stainings), allowing isolation of individual cells by a specific phenotype. Global standard measurements were computed for area

$\left(\mu \mathrm{m}^{2}\right)$, mean fluorescence intensity, perimeter $(\mu \mathrm{m})$, compactness, and cell location (Cartesian coordinates). 2D dot scatterplots were created for each $\mathrm{ROI}$ (region of interest) containing the mean fluorescence intensity of one biomarker on each axis. Using the backgating algorithm, threshold cutoffs were manually positioned to include or exclude cell subpopulations. Total number of cells $\left(\mathrm{DAPI}^{+}\right)$and positively stained cells for CD45, CD3, CD8, and CD15 were segmented in each ROI.

\section{Statistical analysis and reproducibility}

Statistics for $16 \mathrm{~S}$ rRNA gene sequencing, shotgun metagenomic sequencing, and human RNA-seq are described above. Other data were plotted and analyzed using GraphPad Prism 7 for Windows, and specific statistical methods used are indicated in the text or figure legends. Briefly, a two-tailed Mann-Whitney $U$ test was used for comparison between two independent groups. For multi-group comparisons, one-way or two-way ANOVA followed by Dunn's multiple comparisons test was performed. Only statistically significant differences are indicated in the figures. Exact $p$ values and statistical tests used for each panel are reported in the source data. The number of samples analyzed in each group are indicated in each figure.

Sample size of mice follows the $3 \mathrm{Rs}$ (replace, reduce, and refine). Mice were randomly assigned to experimental groups and matched to the best age. No data were excluded from the analyses and 
bioRxiv preprint doi: https://doi.org/10.1101/2021.02.24.432654; this version posted February 27,2021 . The copyright holder for this

preprint (which was not certified by peer review) is the author/funder, who has granted bioRxiv a license to display the preprint in perpetuity. It is made available under aCC-BY-NC-ND 4.0 International license.

932 blinded to group allocation for mouse stool collection and euthanasia to avoid sample cross933 contamination. 
bioRxiv preprint doi: https://doi.org/10.1101/2021.02.24.432654; this version posted February 27, 2021. The copyright holder for this

preprint (which was not certified by peer review) is the author/funder, who has granted bioRxiv a license to display the preprint in perpetuity. It is made available under aCC-BY-NC-ND 4.0 International license.

A.

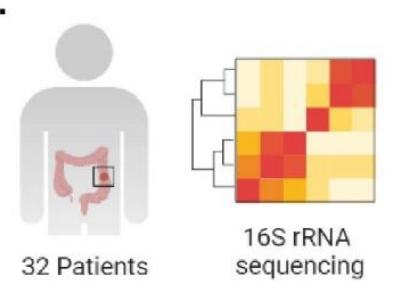

Pathogen vs

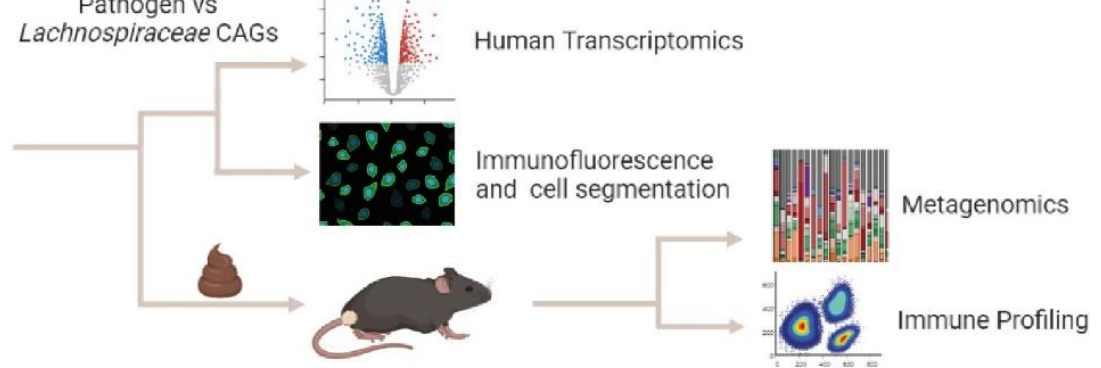

B.

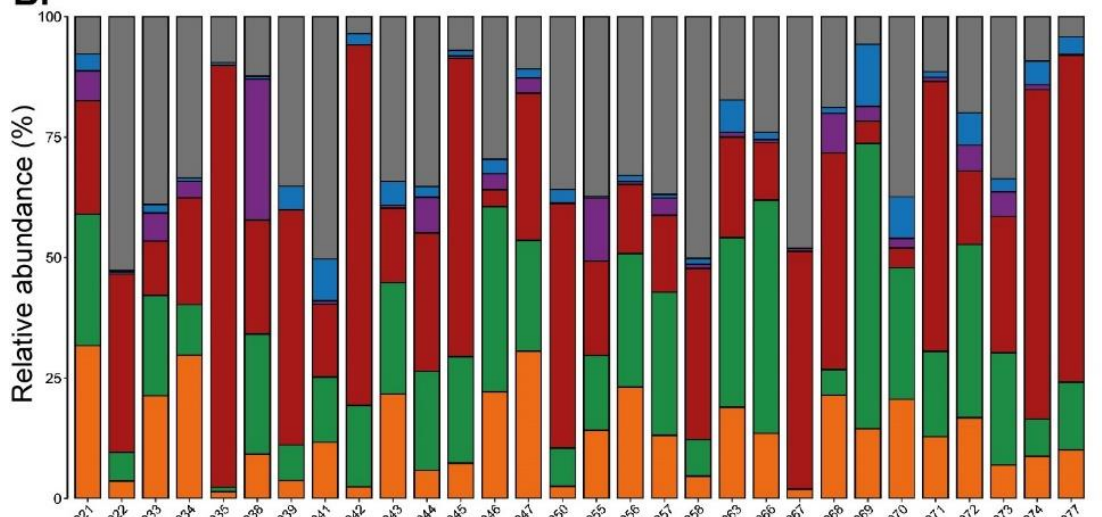

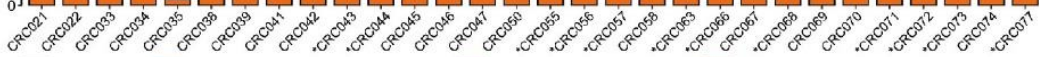
Other Ruminococcus CAG Prevotella CAG Pathogen CAG

Lachnospiraceae CAG Bacteroidetes CAG

D.

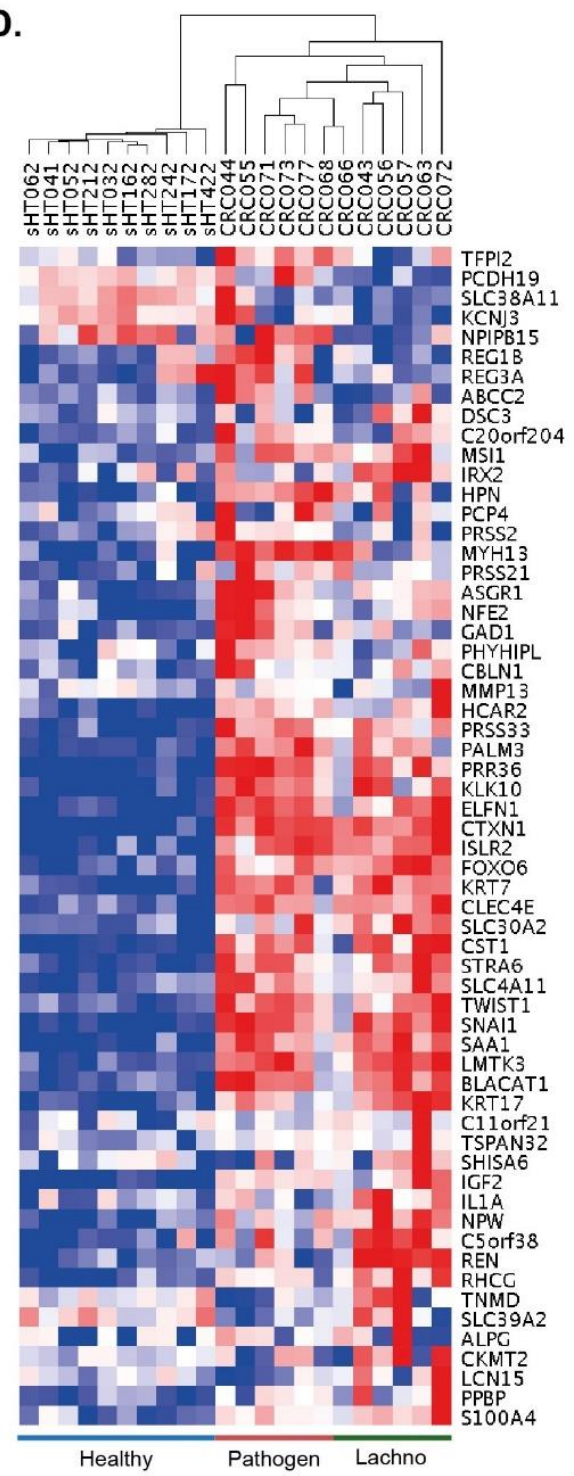

C.

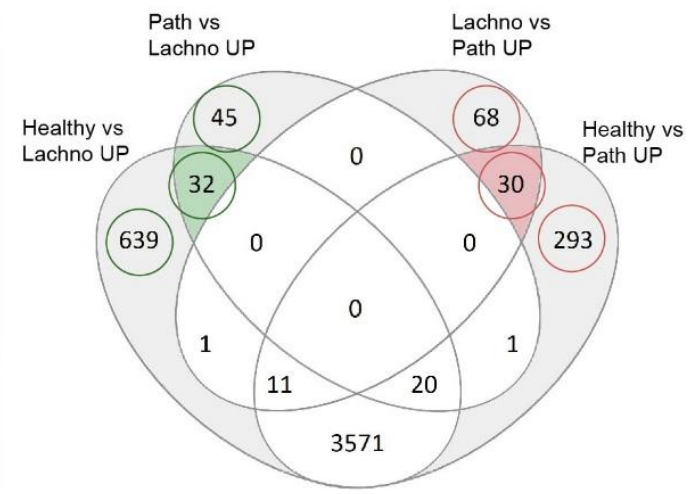

E.

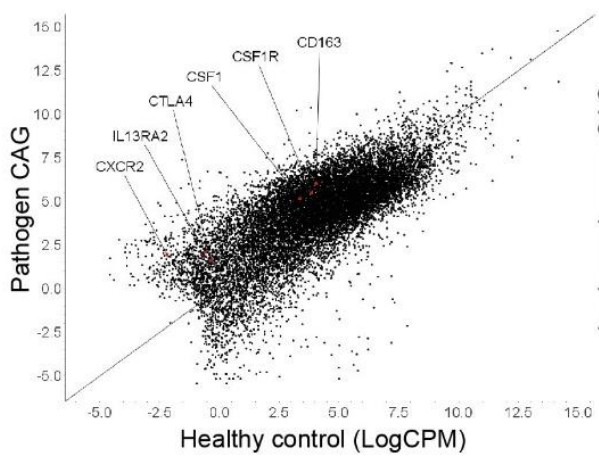

G.

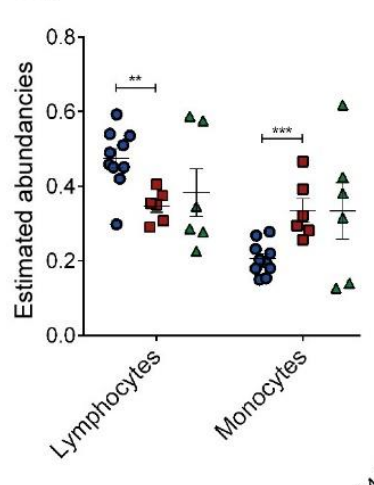

F.

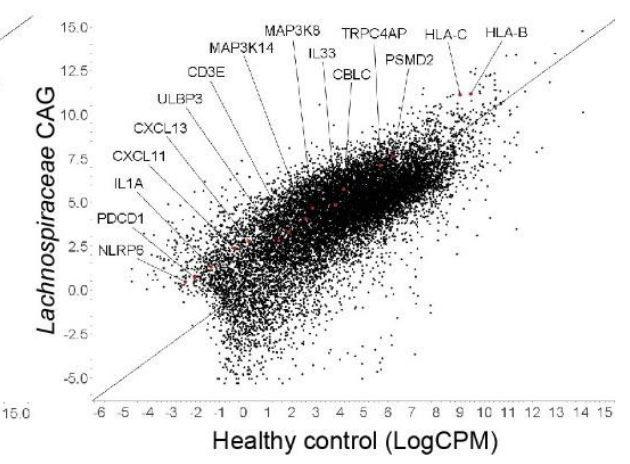

H.

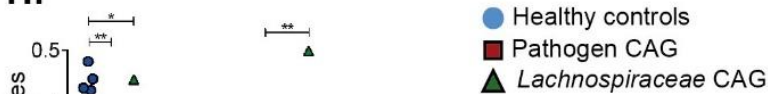

需 $0.4 \%$ 
bioRxiv preprint doi: https://doi.org/10.1101/2021.02.24.432654; this version posted February $27,2021$. The copyright holder for this preprint (which was not certified by peer review) is the author/funder, who has granted bioRxiv a license to display the preprint in perpetuity. It is made available under aCC-BY-NC-ND 4.0 International license.

Figure 1. Distinct microbiomes in patients with adenomas and CRC correlate with differential human immune transcripts

A. Overview of the experimental design. 32 treatment-naïve patients were included in the study. Surgical resections were collected from multiple sites in the colon and analyzed by 16S rRNA sequencing, immunofluorescence, and RNA expression analysis. Selected fecal samples were collected anaerobically and administered in a germ-free cancer mouse model. B. Human microbiota composition measured by proportional abundance of bacterial CAGs in human colon biopsies. CAGs are named after the most abundant genus. Stars $\left.{ }^{*}\right)$ indicate the 12 patients selected for bulk RNAseq analysis. C. Venn diagram depicting numbers of significantly DEGs ( $p$ value $<0.5$ ) between healthy controls (Healthy, $n=10$ ), tumors from the Pathogen CAG (Path, $n=6$ ), and tumors from subjects harboring the Lachnospiraceae CAG (Lachno, $n=6$ ). The gene numbers circled in red are those uniquely elevated in the Pathogen CAG and gene numbers circled in green are uniquely elevated in the Lachnospiraceae CAG. D. Heatmap of unsupervised hierarchal clustering of genes and patients, representing the top 60 significantly DEGs (CRC versus healthy controls; FDR adjusted $p$ value $<0.1$, and Lachnospiraceae CAG versus Pathogen CAG; $p$ value $<0.05$. All $\log _{2} F C \leq-1.5$ and $\geq 1.5$ ), consisting of the top 10 significantly DEGs from each circle highlighted in the Venn diagram (panel C). E, F. Expression plots displaying labeled immune genes differentially expressed and uniquely elevated in (E) Pathogen CAG and (F) Lachnospiraceae CAG tumors relative to healthy controls. The $\mathrm{x}$-axis is the logCPM values for healthy controls and the $y$-axis is the logCPM values for (E) Pathogen CAG and (F) Lachnospiraceae CAG tumors. Red dots show genes of interest. G, H. Estimated immune cell abundancies from whole transcriptomic data deconvoluted with the CIBERSORTx software in healthy controls and tumors. Estimated lymphocyte abundances were calculated as the sum of proportions of naïve B cells, memory B cells, CD8 ${ }^{+} T$ cells, naïve CD4 ${ }^{+} T$ cells, resting memory $\mathrm{CD}^{+} \mathrm{T}$ cells, and activated memory $\mathrm{CD} 4^{+} \mathrm{T}$ cells. Estimated monocyte abundances were calculated as the sum of proportions of monocytes, M0 macrophages, M1 macrophages, and M2 macrophages. Bars represent mean \pm SEM. $p$ values were calculated by unpaired Student's t-tests. ${ }^{*} p \leq 0.05,{ }^{* *} p \leq 0.01,{ }^{* * *} p \leq$ $0.001,{ }^{* * * *} p \leq 0.0001$. 
A.

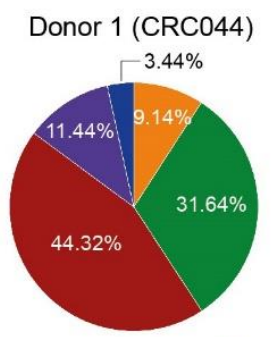

Donor 2 (CRC056)

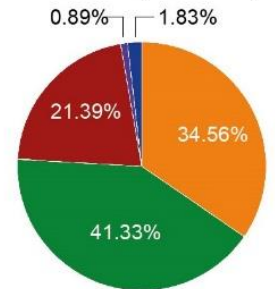

Ruminococcus CAG —Prevotella CAG — Pathogen CAG Lachnospiraceae CAG $\square$ Bacteroidetes CAG

C.

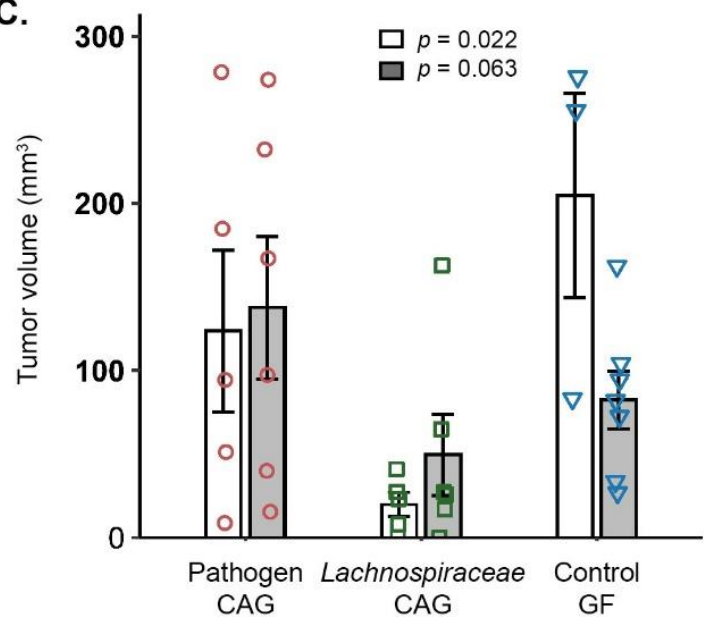

E.

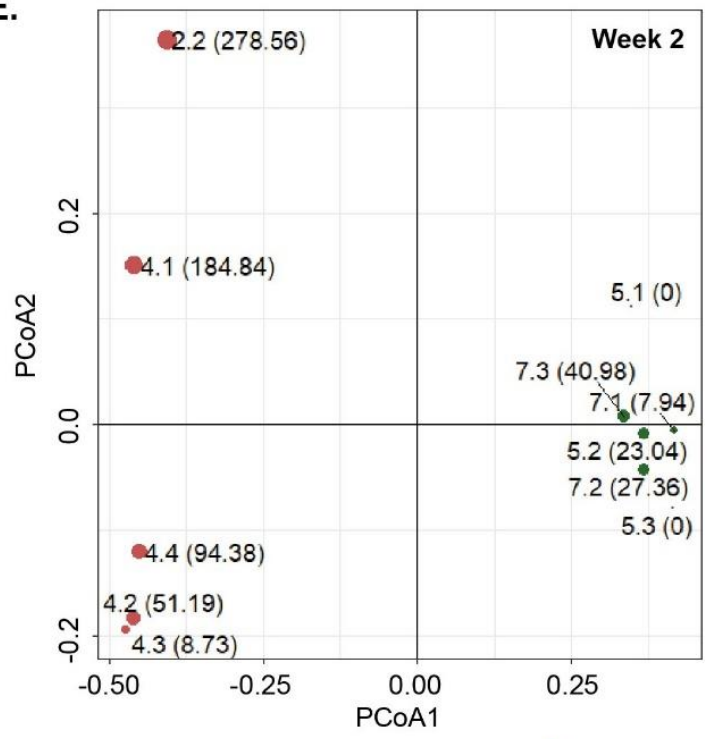

B.
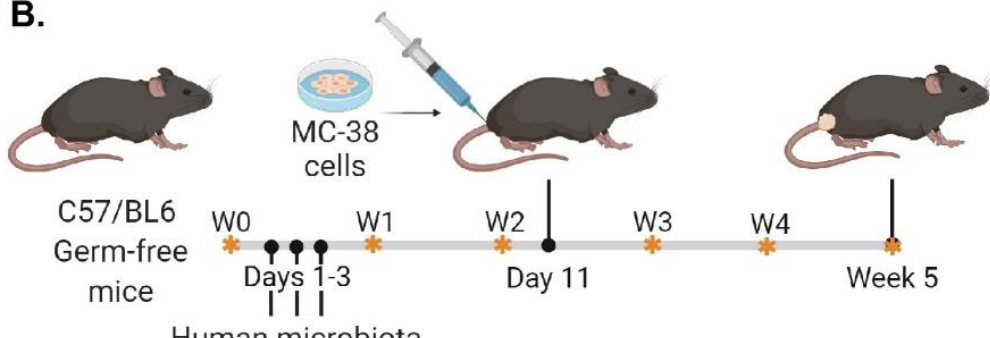

Human microbiota administration

D.
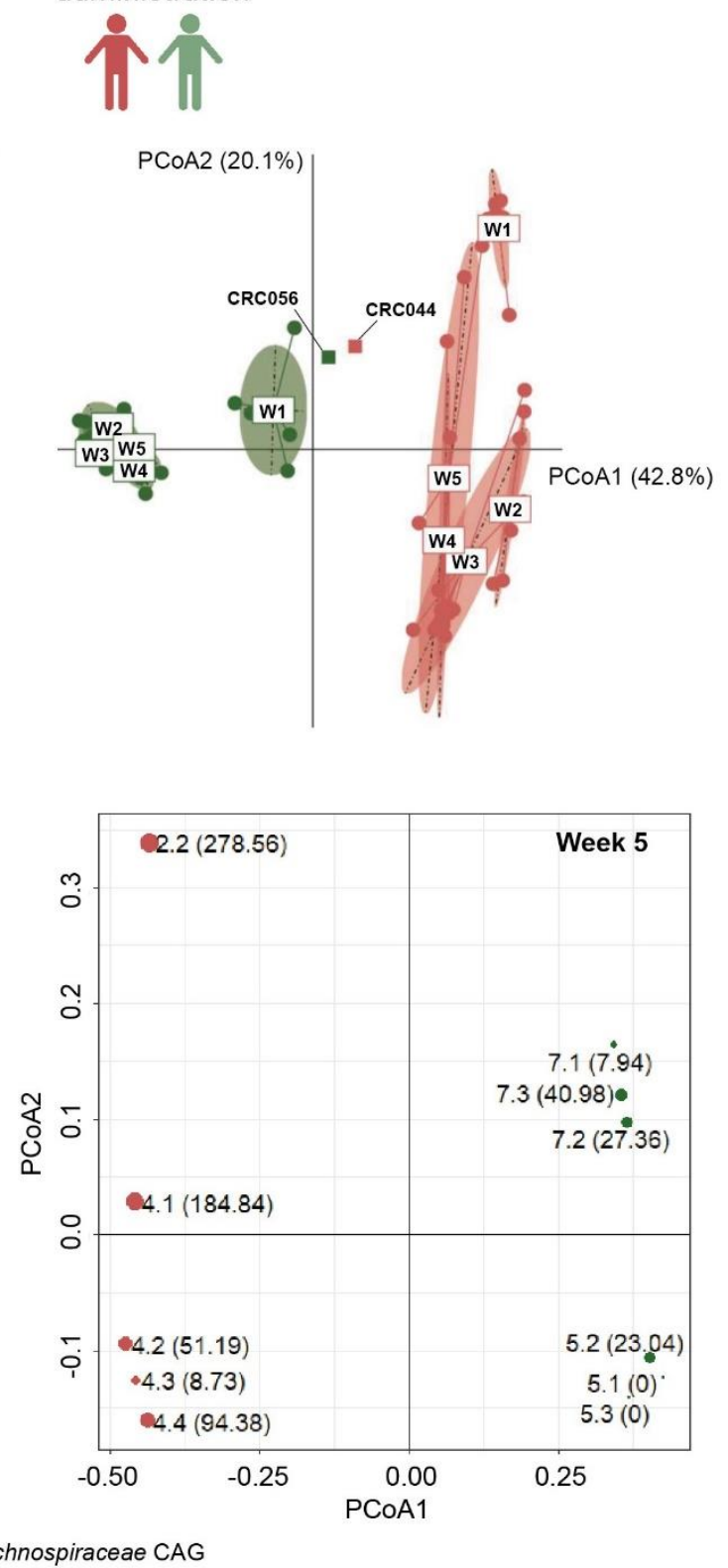

Figure 2. Tumor growth is strongly dependent on microbiota type in a humanized mouse model of CRC.

A. Validation of microbiota composition in human donors for the pre-clinical mouse trial. Colon resections were collected at surgery and the mucosal microbiota was profiled using 16S rRNA sequencing. Pie-charts represent the abundance of the five bacterial CAGs on adenoma or tumor samples from each donor. Donor 1 (CRC044), diagnosed with a T3N2 rectum adenocarcinoma, was selected based on the high abundance of a Pathogen CAG microbiota and Donor 2 (CRC056), diagnosed with a tubulovillous adenoma, was selected based on the high abundance of a Lachnospiraceae CAG microbiota. B. Experimental design of the pre-clinical trial with a humanized MC-38 model of CRC. C. Tumor growth is reduced in mice receiving the Lachnospiraceae 
970 microbiota compared to mice receiving the Pathogen CAG microbiota or control germ-free (GF) mice. Tumor 971 volume was measured with a caliper at endpoint and calculated as (length $\mathrm{x}$ width $\left.{ }^{2}\right) / 2$. Overall $p$ values were 972 calculated with the Kruskal-Wallis test. Data indicate mean \pm SEM. $n=3-6$ replicates/group per condition. Data 973 from two independent experiments are shown by open and grey bars. D. Relatedness ( $\beta$-diversity) of the fecal 974 microbiota of the two human donors and respective recipient mice at different time-points represented by 975 principal coordinate analysis $(\mathrm{PCOA})$ on Bray-Curtis distance matrix (PERMANOVA $r^{2}=0.79 ; p$ value $=0.001$ ). 976 W, week. E PCoA plots of the Metaphlan2 species-level taxa profiles of the murine fecal microbiomes, 977 performed at week 2 (left) and week 5 (right). Fecal microbiome profiles corresponding to the Pathogen CAG 978 donor and the Lachnospiraceae CAG donor are colored in red and green, respectively. Each point corresponds 979 to a specific mouse ID and the corresponding tumor volume is shown within parentheses. The size of each point 980 is proportional to the tumor volume. PERMANOVA $r^{2}=0.24 ; p=0.016$ (week 2 ), $\mathrm{r}^{2}=0.25 ; p$ value $=0.006$ (week $9815)$. 
bioRxiv preprint doi: https://doi.org/10.1101/2021.02.24.432654; this version posted February 27, 2021. The copyright holder for this

preprint (which was not certified by peer review) is the author/funder, who has granted bioRxiv a license to display the preprint in perpetuity. It is made available under aCC-BY-NC-ND 4.0 International license.
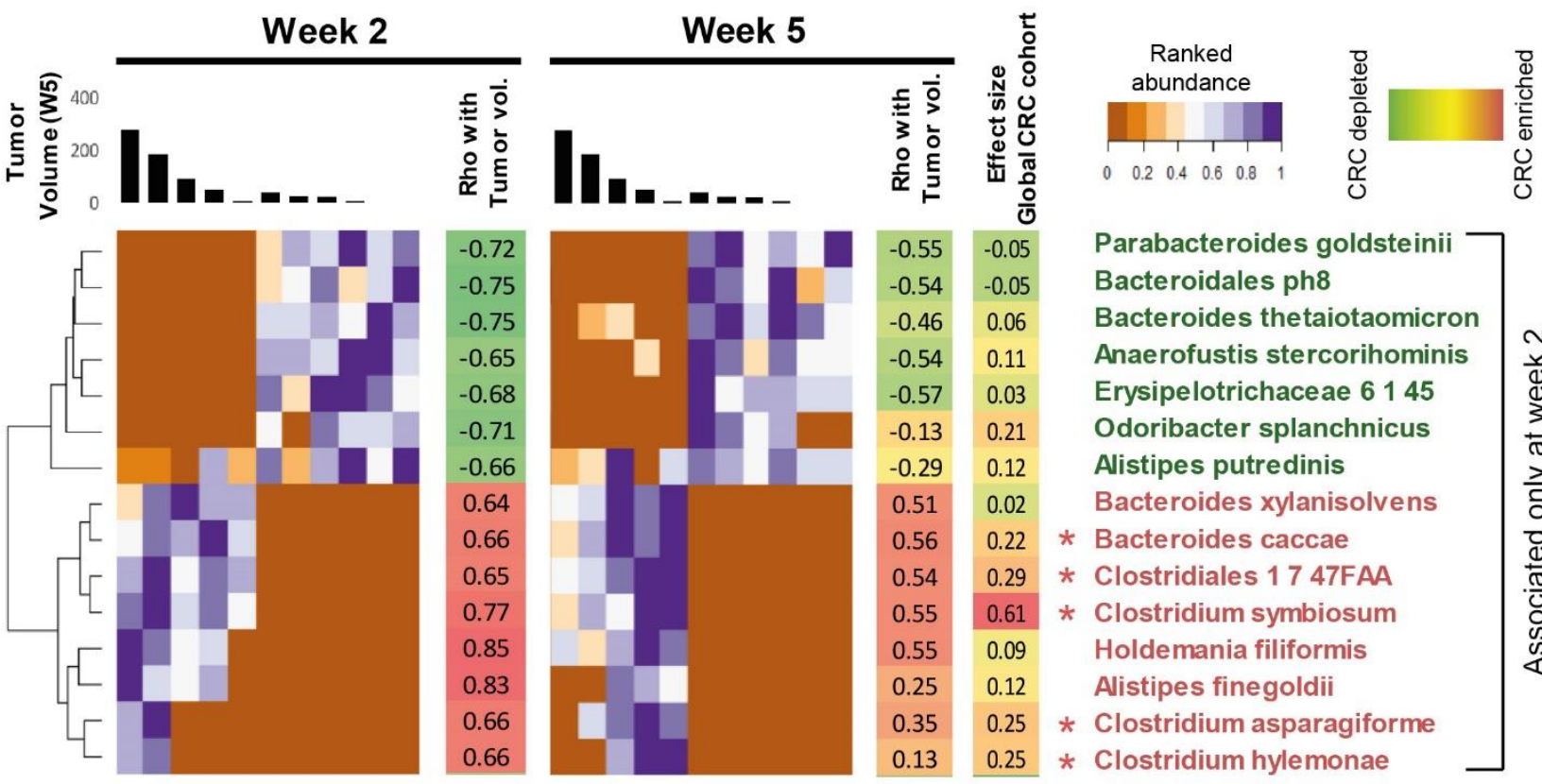

Parabacteroides goldsteinii Bacteroidales ph8

Bacteroides thetaiotaomicron

Anaerofustis stercorihominis

Erysipelotrichaceae 6145

Odoribacter splanchnicus

Alistipes putredinis

Bacteroides xylanisolvens

* Bacteroides caccae

* Clostridiales 17 47FAA

* Clostridium symbiosum

Holdemania filiformis

Alistipes finegoldii

* Clostridium asparagiforme

* Clostridium hylemonae
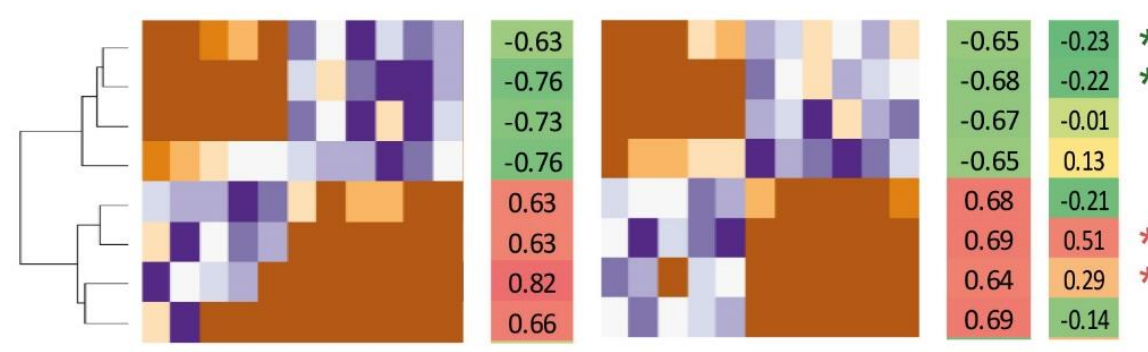

$\begin{aligned} \text { * } & \text { Coprococcus comes } \\ \text { * } & \text { Ruminococcus lactaris } \\ & \text { Bacteroides finegoldii } \\ & \text { Erysipelotrichaceae } 2244 A \\ & \text { Ruminococcus obeum } \\ \text { * } & \text { Clostridium hathewayi } \\ \text { * } & \text { Flavonifractor plautii } \\ & \text { Coprococcus sp ART55 } 1\end{aligned}$
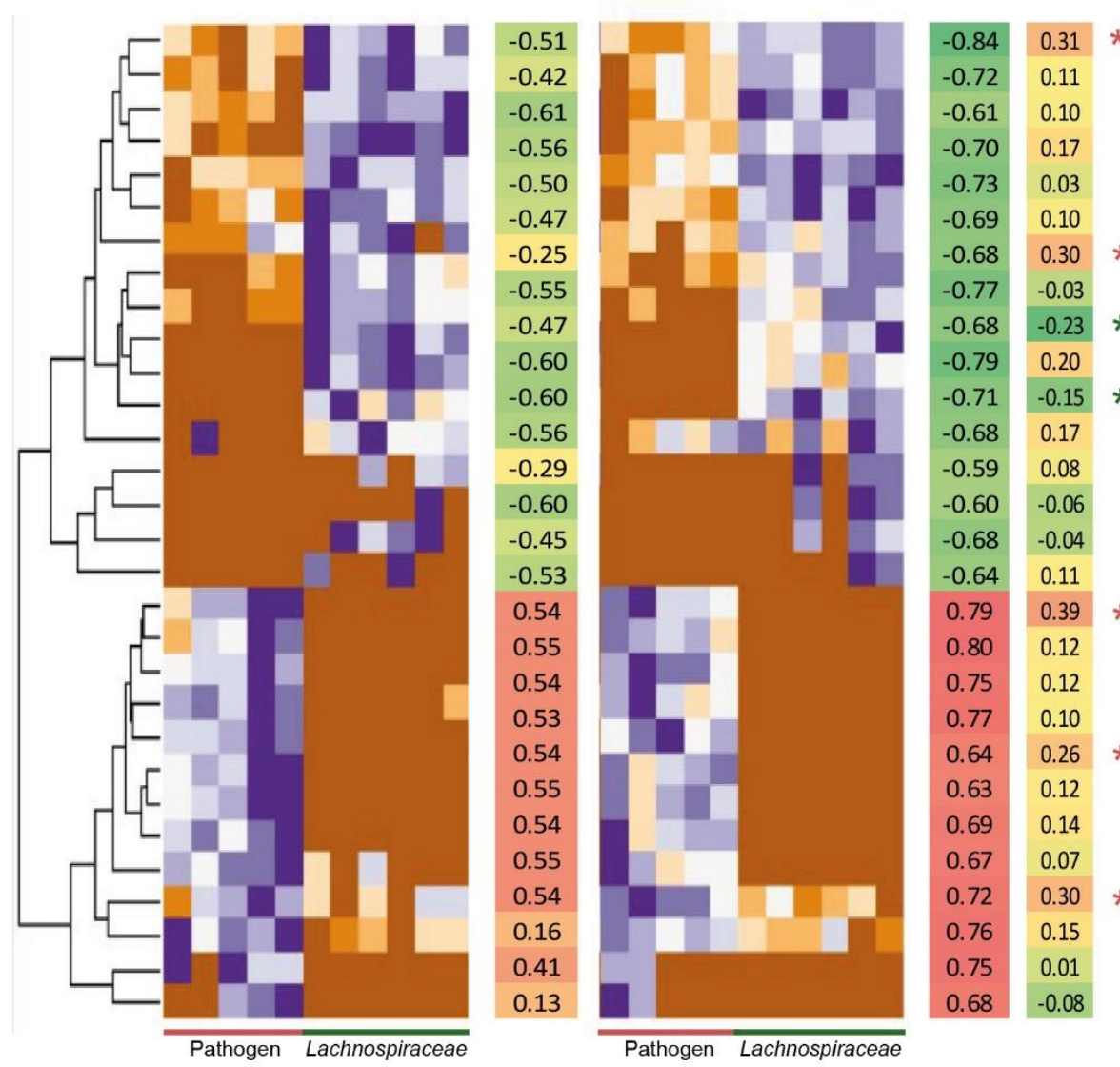

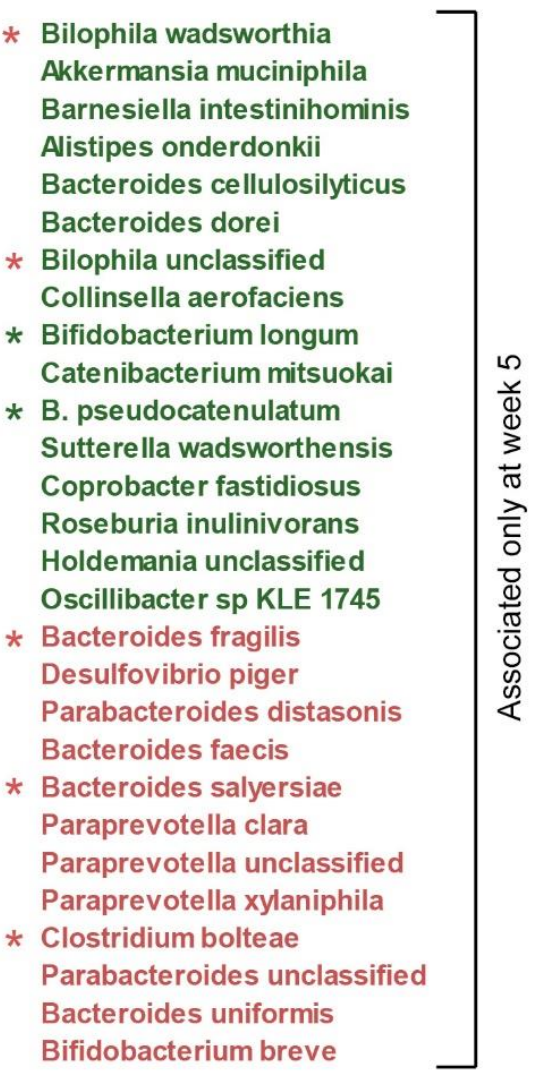


bioRxiv preprint doi: https://doi.org/10.1101/2021.02.24.432654; this version posted February $27,2021$. The copyright holder for this preprint (which was not certified by peer review) is the author/funder, who has granted bioRxiv a license to display the preprint in perpetuity. It is made available under aCC-BY-NC-ND 4.0 International license.

983 Figure 3. Distinct bacterial taxa are associated with final tumor volume

984 Heatmap showing the ranked abundances of 'tumor positive' (red) and 'tumor negative' (green) taxa in mice 985 fecal microbiomes at week 2 only (top panel), shared between week 2 and 5 (middle panel), and week 5 only 986 (bottom panel), as determined by shallow shotgun sequencing of samples from experiment 2. For each mouse, 987 the corresponding tumor volume at week 5 is indicated as bar plots at the top of the heatmap. Spearman's rank 988 correlations between bacterial taxa abundance at different time points and the tumor volume at week 5 are 989 indicated. The effect sizes (Cohen's D) observed for the various taxa in the Global Reference cohort are also 990 shown. Taxa that are significantly enriched or depleted in CRC $(n=325)$ versus healthy individuals $(n=310)$ 991 (identified using Mann-Whitney $U$ tests, $p$ values corrected using Benjamini-Hochberg for FDR $<0.1$ ) are 992 indicated by red and green stars, respectively. Positive association with tumor volume and enrichment in CRC 993 in the Global Reference cohort is color-coded in red. Negative association with tumor volume and depletion in 994 CRC in the Global Reference cohort is color-coded in green. 
A.

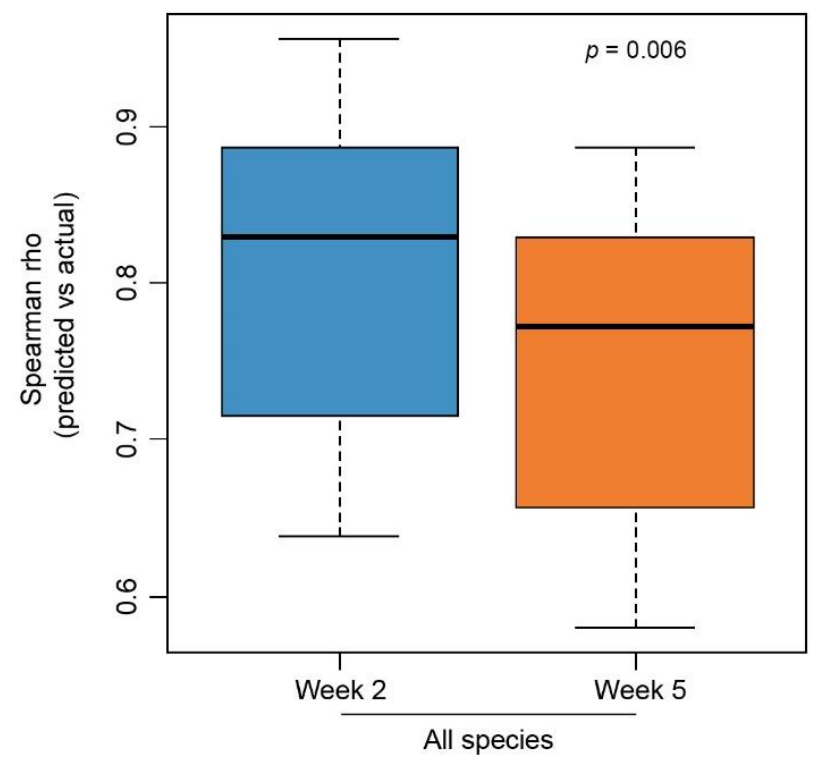

B.

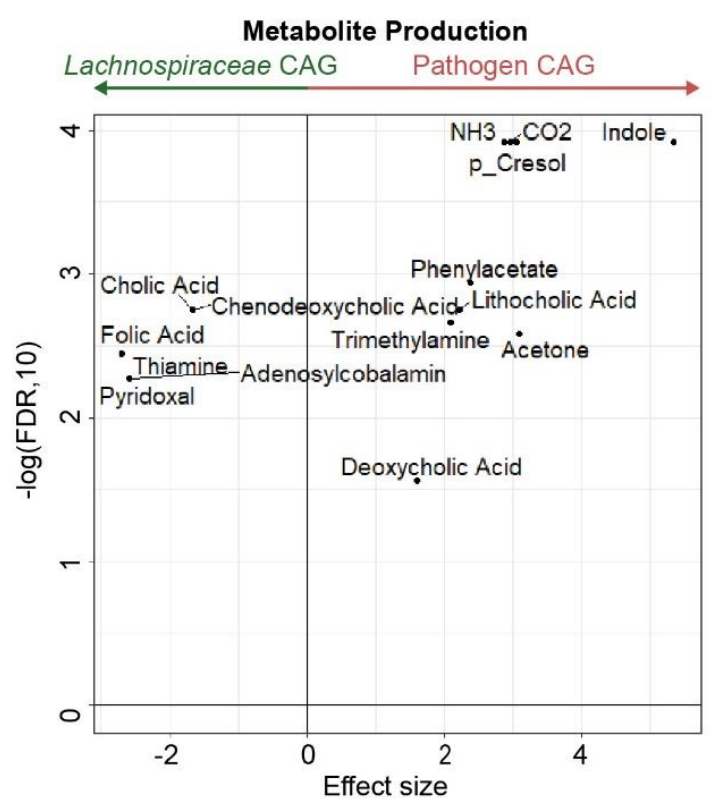

C.

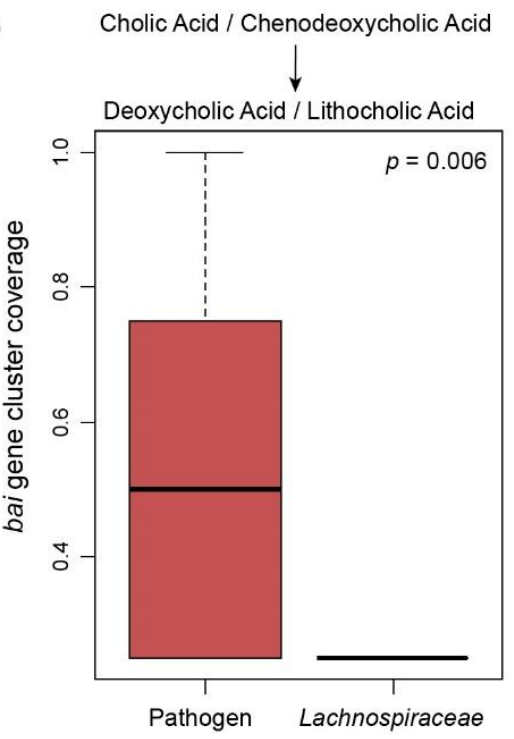

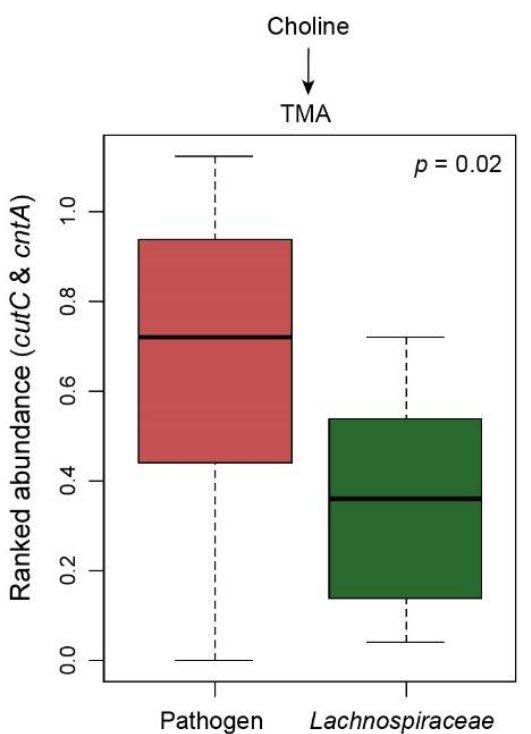

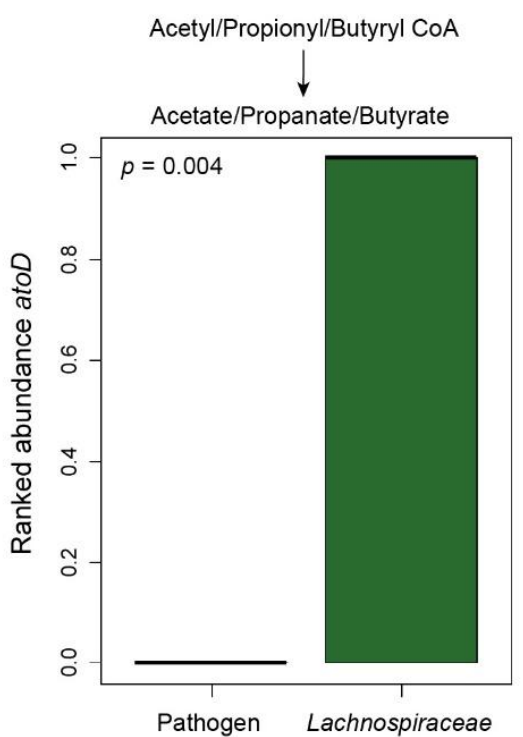

\section{Figure 4. High-risk and low-risk microbiome are associated with different metabolic pathways}

A. Pre-tumor microbiota is predictive of tumor growth. Tumor-associated bacterial taxa at week 2 have higher predictability for tumor volume than taxa at week 5. Boxplots show the variation of Spearman rho values calculated between the predicted and actual tumor volumes obtained for the 100 iterations of the two variants of RF models (trained on week 2 and week 5 abundance profiles, respectively). Mann-Whitney $U$ test $p$ values for the different comparisons are indicated. B. Volcano plot showing the validated set (identified as summarized in Fig. S13) of metabolite production functionalities that were predicted to have either a significant positive or negative association with the Pathogen CAG microbiome. The $x$-axis indicates the effect size difference (negative indicating enriched in the Lachnospiraceae CAG and positive indicating enriched in the Pathogen CAG), and the $y$-axis indicates the negative log of FDR value. C. Boxplots comparing (left) the coverage of the bile acid inducible (bal) gene cluster that converts the primary bile acids (cholic acid and chenodeoxycholic acid) into secondary bile acids (deoxycholic acid and lithocholic acid); (middle) the cumulated gene abundances of CntA and CutA enzymes that catalyze trimethylamine (TMA) production; and, (right) the abundance of the AtoD enzyme catalyzing the last step of short-chain fatty acids formation, between the Pathogen and the Lachnospiraceae microbiome types. The $p$ values obtained using the Mann-Whitney $U$ tests are indicated. 
A.
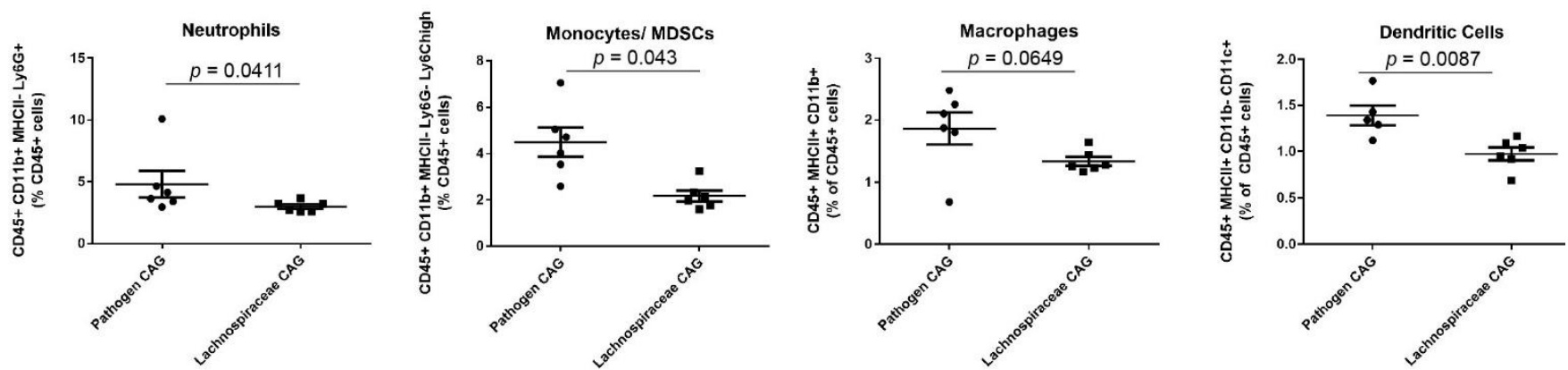

B.
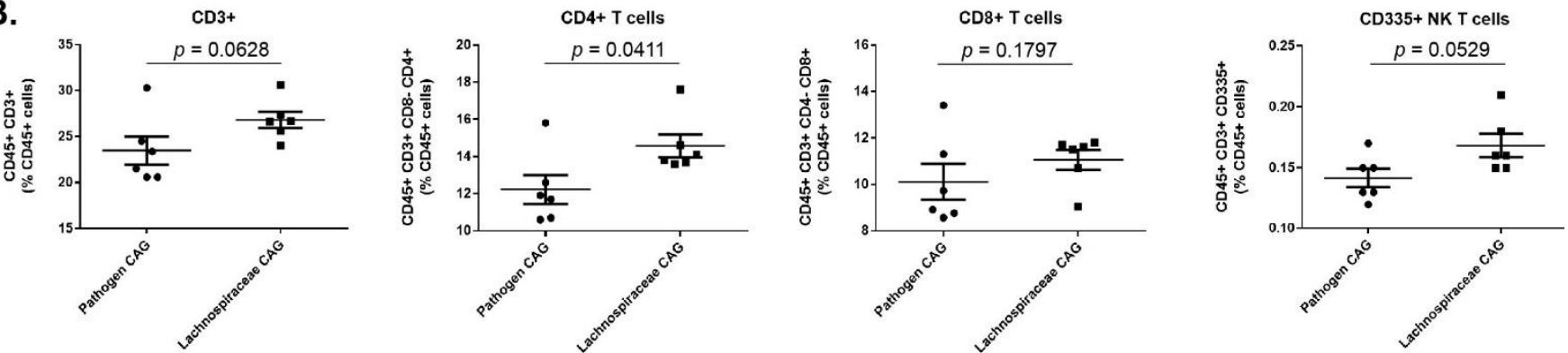

C.

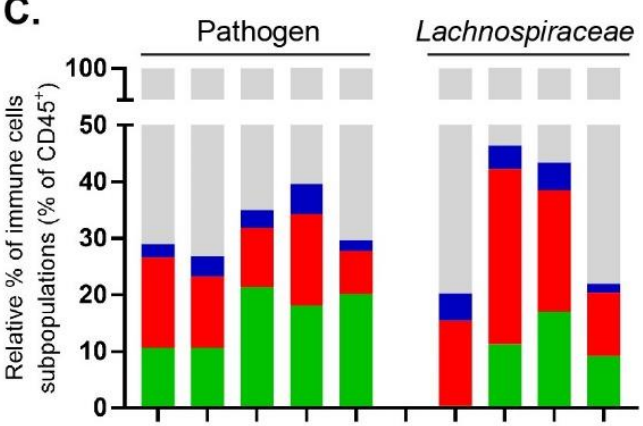

D.

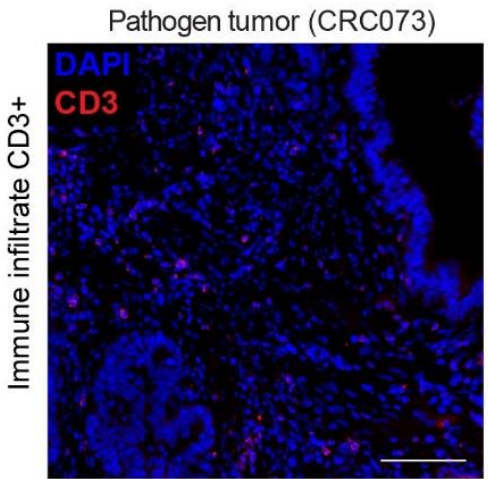

Lachnospiraceae tumor (CRC057)
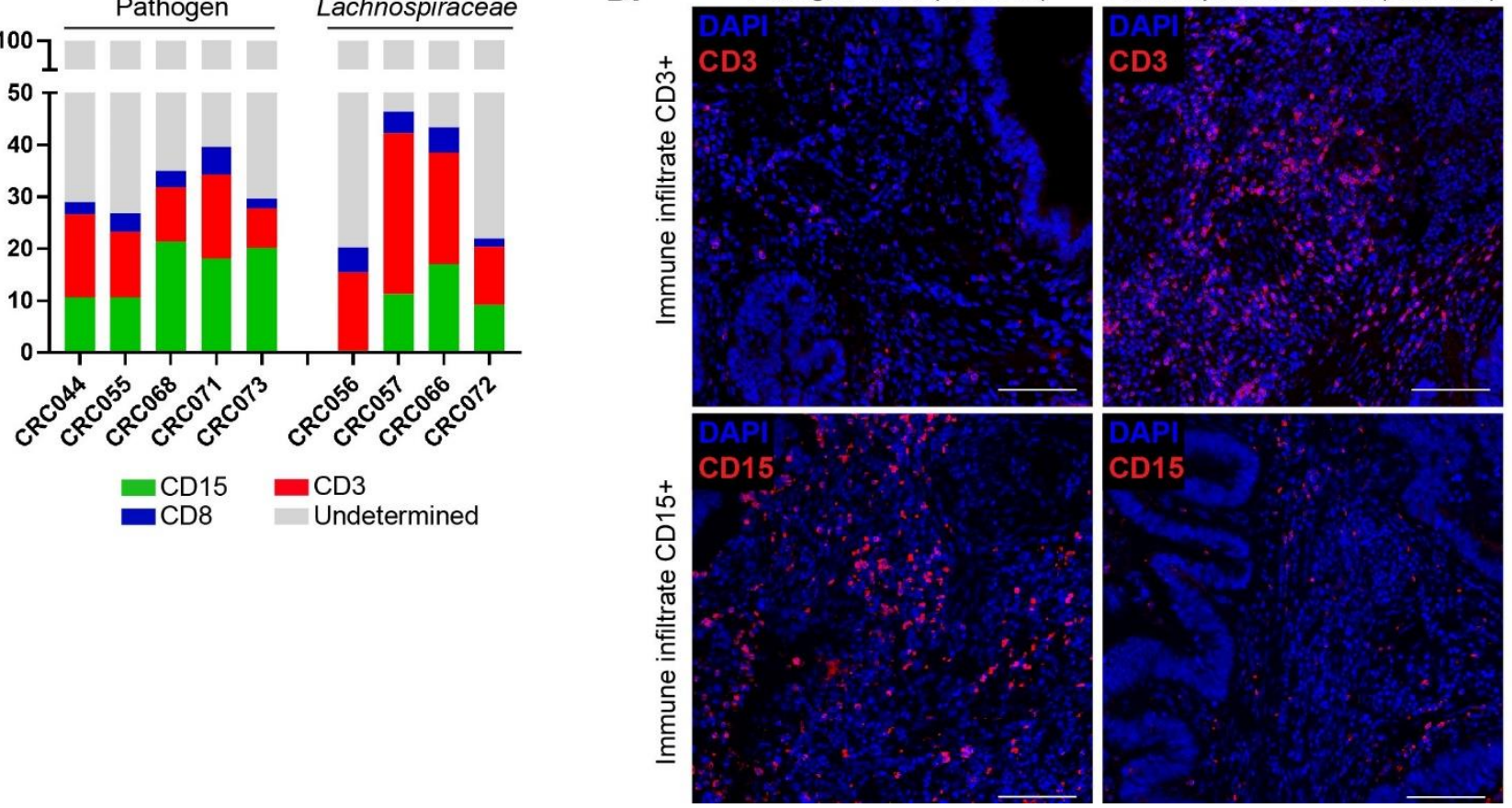

Figure 5. Lachnospiraceae-type microbiome colonization induces a strong immune infiltration and antitumoral immune response.

A. Spleens from mice with the Pathogen CAG have more neutrophils (CD45+CD11b+MHCII-Ly6G+Ly6low), monocytes $\left(\mathrm{CD} 45^{+} \mathrm{CD} 11 \mathrm{~b}+\mathrm{MHCll}-\right.$ Ly6G-LyChigh), macrophages $\left(\mathrm{CD} 45^{+} \mathrm{CD} 11 \mathrm{~b}+\mathrm{MHCl}+\mathrm{I}^{+}\right)$, and dendritic cells $\left(\mathrm{CD} 45^{+} \mathrm{MHCll}+\mathrm{CD} 11 \mathrm{~b}-\mathrm{CD} 11 \mathrm{C}^{+}\right)$, as determined by flow cytometry gated on $\mathrm{CD}^{2} 5^{+}$cells. Panels show quantification of neutrophils, monocytes, macrophages, and dendritic cells.

B. Spleens from mice with the Lachnospiraceae CAG have more $\mathrm{CD}^{+}, \mathrm{CD}^{+}, \mathrm{CD} 8^{+}$, and NK T cells, as determined by flow cytometry gated on $\mathrm{CD}_{45^{+}}$cells. $p$ values were determined by Mann-Whitney $U$ test and are represented in each plot. Data indicate mean \pm SEM. $n=6$ biological replicates/group. C. Quantification of immune infiltrate $\left(C D 45^{+}\right), T$-cell infiltrate $\left(\mathrm{CD}^{+}\right.$and $\left.\mathrm{CD}^{+}\right)$and neutrophil infiltrate $\left(\mathrm{CD15^{+ }}\right)$ in human $\mathrm{CRC}$ biopsies from Pathogen $(n=5)$ and Lachnospiraceae-enriched tumors $(n=4) .2$ sections/tumor and 3 ROls quantified per section (same ROls were 
bioRxiv preprint doi: https://doi.org/10.1101/2021.02.24.432654; this version posted February 27,2021 . The copyright holder for this

preprint (which was not certified by peer review) is the author/funder, who has granted bioRxiv a license to display the preprint in perpetuity. It is made available under aCC-BY-NC-ND 4.0 International license.

1025 used to quantify different immune cell subpopulation in each tumor), means are shown, group comparison with 1026 one-way ANOVA. D. Immunofluorescence representative images of Pathogen CAG-enriched tumors (CRC073) 1027 and Lachnospiraceae CAG-enriched tumors (CRC057) human tumors showing that more $\mathrm{CD}^{+} \mathrm{T}$ cells (red) 1028 infiltrate into Lachnospiraceae tumors, while more CD15+ neutrophils infiltrate into Pathogen tumors. 1029 Counterstained with nuclear dye DAPI. All tumors were analyzed and for each tumor; 3 ROls were quantified 1030 per section ( $\mathrm{n}=2$ sections/tumor/staining). Scale bars $100 \mu \mathrm{m}$. 
Table 1 - Clinico-pathological characteristics of the two donors.

\begin{tabular}{|c|c|c|}
\hline & CRC044 & CRC056 \\
\hline Gender & Female & Male \\
\hline $\begin{array}{l}\text { Age (years, at } \\
\text { the time of } \\
\text { surgery) }\end{array}$ & 66 & 65 \\
\hline Tumor Type & Adenocarcinoma & $\begin{array}{l}\text { Tubulovillous adenoma with low } \\
\text { grade dysplasia }\end{array}$ \\
\hline $\begin{array}{l}\text { Tumor } \\
\text { Location }\end{array}$ & Rectum & Hepatic flexure \\
\hline $\begin{array}{l}\text { Tumor Size } \\
(\mathrm{cm})\end{array}$ & 3.5 & - \\
\hline T-category & T3 & T0 \\
\hline $\mathrm{N}$-category & $\mathrm{N} 2 \mathrm{~b}$ & - \\
\hline BMI & $\mathrm{N} / \mathrm{A}$ & 25.7 \\
\hline Hip/waist ratio & $\mathrm{N} / \mathrm{A}$ & 0.98 \\
\hline Medication & Bendnoflumethiazole 5mgs OD & $\begin{array}{l}\text { Atrovasin 20mg OD, Eltroxin 50mgs } \\
\text { OD }\end{array}$ \\
\hline $\begin{array}{c}\text { Bowel } \\
\text { Preparation }\end{array}$ & $\begin{array}{l}\text { Klean Prep } \times 1 \text { and Picolax ( } 5 \text { days } \\
\text { prior to surgery) followed by low } \\
\text { residue diet } \times 5 \text { days and Phosphate } \\
\text { enema } \times 2 \text { on morning of the } \\
\text { surgery }\end{array}$ & $\begin{array}{l}\text { Klean Prep } \times 1 \text { and Picolax ( } 5 \text { days } \\
\text { prior to surgery) followed by low } \\
\text { residue diet } \times 5 \text { days and Phosphate } \\
\text { enema } \times 2 \text { on morning of the } \\
\text { surgery }\end{array}$ \\
\hline $\begin{array}{l}\text { 3-year clinical } \\
\text { folow-up }\end{array}$ & $\begin{array}{l}\text { Patient had adjuvant chemotherapy } \\
\text { following surgery. Patient finished } \\
\text { oral Capecitabine in October } 2017 \\
\text { and a CT scan in January } 2018 \\
\text { which showed no evidence of } \\
\text { disease. Development of symptoms } \\
\text { (dysphasia and right-sided facial } \\
\text { droop) in February } 2020.18 \mathrm{~mm} \\
\text { brain metastases consistent with } \\
\text { rectal cancer. Negative CT scan } \\
\text { and negative colonoscopy in June } \\
2020 \text {. }\end{array}$ & $\begin{array}{l}\text { No further treatment required to } \\
\text { date }\end{array}$ \\
\hline
\end{tabular}

